\title{
Aplicación práctica de técnicas docentes para Contabilidad Financiera
}

Practical application of alternative teaching methods in Accounting

José Luis Beltrán Varandelaa (jilbeltran@uvigo.es)

José Manuel Pereirab (jpereira@ipca.pt)

José Luis Sáez Ocejoa (jocejo@uvigo.es)

aUniversidad de Vigo (España), blnstituto Politécnico do Cávado e do Ave (Portugal)

http://dx.doi.org/10.12795/EDUCADE.2011.i02.02

RESUMEN: El objeto del trabajo consiste en estudiar el posible grado de interés, eficacia y eficiencia de las estrategias docentes: "técnicas del papel al minuto", la realización de "trabajos resumen" y "trabajos en equipo" en orden a valorar el nivel de satisfacción, utilidad y sugerencias de mejora que los estudiantes percibieron al aplicárseles dichas metodologías, en base a una experiencia docente realizada en un curso de $1^{\circ}$ de la asignatura Contabilidad Financiera de la Diplomatura de Ciencias Empresariales de la Universidad de Vigo. El análisis se circunscribe en el nuevo entorno futuro de la Declaración de Bolonia, a través del procedimiento de la encuesta entre los alumnos que participaron. Asimismo se evalúa el rendimiento de los alumnos en función de sus resultados en la convocatoria de junio de 2010. Los resultados obtenidos permiten observar el nivel de satisfacción y utilidad de las técnicas, a pesar de ello se observan pocas diferencias entre aquellos alumnos que habían superado la asignatura usando dichas estrategias de aprendizaje, con respecto a los que no las usaron

PALABRAS CLAVE: Técnicas docentes, Papel al minuto, Trabajo en grupo, Estrategias organizativas: Resúmenes, Docencia en Contabilidad Financiera.

\begin{abstract}
The purpose of this paper is to study the possible degree of interest, efficiency and effectiveness of teaching strategies: one -minute papers, groups work and summaries to value the level of satisfaction, usefulness and suggestions for improvement that students perceived on doing these methodologies, based on a teaching course conducted by of the Financial Accounting course Diploma in Business Studies from the University of Vigo.

This analysis is situated within the framework of the Bologna Declaration, through the process of the survey among students who participated. It also evaluates the performance of students based on their findings in the June 2010. The results obtained showed the level of satisfaction and usefulness of the techniques. However, there are few differences between students who passed the subject using these learning strategies with respect to those who did not use
\end{abstract}

KEYWORDS: Classroom assessment techniques, One minute paper, Group work, Organizational strategies: Summaries, Teaching in Financial Accounting.

\section{INTRODUCCIÓN}

Según los objetivos propuestos por la Declaración de Bolonia, dentro del nuevo Espacio Europeo de Enseñanza Superior (en adelante EEES), destaca la idea de la implantación de un proceso de enseñanza continuada durante toda la vida laboral de los titulados universitarios, que va a llevar a un cambio de paradigma en el modelo de enseñanza.

Experiencia docente. Recibido: 01-03-11 - Versiones revisadas: 20-04-11, Aceptado: 30-06-11

Licencia Creative Commons (CC) BY-NC-ND Asociación Española de Contabilidad y Administración de Empresas - AECA 
Para su logro, las metodologías docentes tradicionales, aunque no han quedado obsoletas, van a ser sustituidas por otras que promueven una participación más activa por parte del estudiante.

En España para alcanzar este objetivo, la Comisión para la Renovación de las Metodologías Educativas en la Universidad elaboró un informe sobre "Propuestas para la renovación de las metodologías educativas en la Universidad" (2006). El informe en sus objetivos respecto al proceso de enseñanza-aprendizaje, aboga por un nuevo estilo de trabajo del docente que potencie el uso de nuevas metodologías en el aula. A su vez, añade la necesidad de otorgar mayor protagonismo al alumnado en su aprendizaje, fomentando el trabajo colaborativo y organizando la enseñanza en función de la adquisición, por éstos, de una serie de competencias y potenciando herramientas de aprendizaje autónomo y permanente.

Igualmente el Libro Blanco sobre Estudios de Grado en Economía y Empresa (2005) establece el desarrollo de una serie de competencias, que a la postre persiguen, no sólo que el alumno en contabilidad alcance las competencias específicas básicas, propias de la enseñanza tradicional, sino también otras capacidades como el trabajo en equipo, la adaptación a los continuos cambios de la coyuntura actual, la comprensión de los nuevos fenómenos económicos y la interpretación de la realidad empresarial, todo ello con un desarrollo de la capacidad de reflexión y visión crítica (Arquero, Donoso, Jiménez-Cardoso y González, 2009).

Esta preocupación llevó a la Federación Internacional de Contables (IFAC) a presentar las Normas Internacionales de Educación (IFAC 2009), en las que se indican las habilidades que se esperan de un profesional de la contabilidad, distinguiendo entre habilidades intelectuales (capacidad de resolución de problemas y toma de decisiones), habilidades técnicas (específicas del conocimiento contable), habilidades personales (iniciativa, autoaprendizaje), habilidades interpersonales y de comunicación (trabajo en grupo, presentación, discusión, expresión) y habilidades de organización (liderazgo).

Con este marco de referencia, la consecución de estos objetivos requiere el uso de nuevas metodologías docentes, algunas no tan nuevas y probablemente utilizadas con mayor o menor difusión, que permitan enriquecer las clases favoreciendo la interrelación de conceptos, con el fin de propiciar un mejor seguimiento de la asignatura por parte del alumnado, fomentando el aprendizaje autónomo y cooperativo y una mejoría en los resultados.

Ese fin es el que nos ha llevado a la elaboración de este artículo, en base al uso de una serie de técnicas docentes testadas en la Escuela Universitaria de Estudios Empresariales de Vigo (en adelante EUEE-Vigo), durante el curso 2009-2010, con el fin de su utilización futura en el marco del nuevo "Grado de Comercio" que inicia su andadura en el curso 2010-2011, y más en concreto en la asignatura del actual plan de estudios "Contabilidad Financiera I" de $1^{\circ}$ de la Diplomatura de Ciencias Empresariales, cuya extinción hoy ya es un hecho.

Para ello hemos valorado una experiencia docente sobre el uso de un cóctel de estrategias docentes, en concreto: dos técnicas del "papel al minuto", el uso de metodologías de organización a través de "resúmenes" y el recurso de "trabajos en grupo". La situación de partida sobre la que hemos utilizado estas técnicas es la de valorar la posible situación real con las que nos vamos a encontrar los profesores de la EUEE-Vigo, de cara a las nuevas asignaturas que el nuevo "Grado de Comercio", impartirá en su plan de estudios- "Fundamentos de Contabilidad" y "Contabilidad Financiera", ambas de 6 créditos ECTS- en sustitución a la ya materia extinta Contabilidad Financiera I, con el objetivo de mostrar las ventajas y las dificultades de dichas metodologías de enseñanza, comprobando si las mismas nos permiten obtener 
el grado de excelencia que persiguen las habilidades que se espera de un profesional contable.

\section{TÉCNICAS DOCENTES A APLICAR}

\subsection{PAPEL AL MINUTO}

El uso de la técnica del papel al minuto (one minute paper) es muy reciente en España, tal y como corroboran León Sendra y García Magaldi (2010: 140-141), que la consideran como una estrategia docente útil y que contribuye a mejorar el desarrollo y la adquisición de las competencias transversales o genéricas propuestas en el nuevo modelo universitario europeo. Responden a la denominación genérica de "técnicas del papel al minuto", y su objetivo es el de paliar el problema de entendimiento y falta de atención que tienen los alumnos en las aulas, de modo que puedan mejorar su aprendizaje e incrementar su participación activa en las clases (Rivero, Rivero y Gil, 2000: 1). Estas técnicas son utilizadas como ayuda a la docencia impartida a través de la lección magistral, con el fin de pedir a los alumnos que elaboren un pequeño ejercicio escrito en el que respondan a una serie de conceptos, entre diversas posibilidades, tales como [Rivero, Rivero y Gil, 2000: 5-9); Sáez Ocejo y Rodríguez Rodríguez, 2003: 1-5)]:

1. Realizar un extracto del tema estudiado, destacando los puntos principales tratados, o cuál es la idea central de la lección explicada por el profesor.

2. Poner de manifiesto las dudas o interrogantes que les ha suscitado la lección recibida, o los puntos que todavía quedan sin entender o confusos tras terminar la clase (muddiest point).

3. Contestar en una frase larga y gramaticalmente correcta, cuestiones sobre żquién hace qué, a quién, cuándo, dónde, cómo y por qué? (one sentece summary).

4. Contestar cuestionarios breves, para previamente usarlos al inicio de una nueva lección, o antes de introducir una idea o noción novedosa de cierta importancia (background knowledge probe).

5. La variante de la anterior, orientada hacia nociones que dificulten el aprendizaje del estudiante (misconception /preconception check).

6. Resumir, correctamente redactada, la idea clave que se ha expuesto en la sesión impartida previamente (directed paraphrasing).

Estas técnicas pedagógicas se emplean con el fin de evaluar la dinámica de la clase y observar resultados y posibilidades en el alumnado (Chizmar y Ostrosky, 1998:7). De todas las anteriores, aplicaremos una variante mixta de las técnicas background knowledge probel y misconception /preconception check2, pero aplicada a posteriori, basada en la realización de una serie de preguntas de respuesta corta a los

\footnotetext{
1 Con la técnica del "Background Knowledge Probe", se exige al estudiante que conteste de forma breve, con una palabra o frase, o que elija la respuesta correcta entre varias posibles tipo test -. Con este sistema se pretende que el alumno conteste lo que piensa y sabe, para ayudar al instructor a tomar decisiones eficaces sobre el modo de enfocar la clase, para un buen entendimiento de lo que se trata de comunicar en el aula y sobre los contenidos impartidos.

${ }^{2}$ La técnica misconception/preconception check puede ser particularmente útil en materias que traten de cuestiones que puedan generar dudas, en las que los alumnos puedan no haber entendido el concepto o haberlo desarrollado de forma errónea. En ese sentido, el docente ha de valorar, qué conceptos pueden ser menos entendidos por parte de los estudiantes, de manera que ellos mismos conozcan las limitaciones de algunos conocimientos, o la imprecisión de ciertos conceptos, mediante la discusión y comparación con otra serie de cuestiones y respuestas, produciéndose la oportunidad de un adicional feedback y una autocorrección.
} 
estudiantes, al objeto de comprobar su eficacia y grado de utilidad. A su vez, y dentro del mismo enfoque se usó otra variante, en concreto el recurso-técnica muddiest point 3

Estos métodos presentan muchas ventajas, ya que requieren poco tiempo de preparación por parte del docente, al ser un método rápido, simple y fácil de organizar, proporcionando un contacto directo con el alumnado, ya que suministra un método alternativo de contestación a las dudas surgidas por aquellos alumnos que no les gusta hacer preguntas en clase (Cross y Angelo, 1993: 154-158). Por otra parte, al docente, le proporciona instantáneamente las posibles dificultades sobre los conocimientos impartidos, junto con una perspectiva del nivel de la clase.

La literatura sobre el estado de la cuestión, nos indica que todas las experiencias docentes han tenido resultados positivos, por los beneficios que aporta tanto al profesorado como a los alumnos, tal y como refutan los resultados de Stead (2005: 5) o Moreno, Ibáñez y Gisbert (2009: 7), aunque éstos recomiendan su uso en clases prácticas, y para grupos pequeños, esta técnica puede ser útil para cualquier número de alumnos. Froyd y Layne (2008) conciben el one minute paper, como una forma rápida y sencilla de evaluar, cuándo y dónde comenzaron a perderse nuestros alumnos, proponiéndola como un instrumento útil de complemento y término de la lección magistral Cantillon (2003).

Desde el punto de vista del alumno, las estrategias contribuyen a mejorar el clima de la clase favoreciendo de manera natural una interacción profesor-alumno, que de otra manera no se hubiera producido Lucas, (2010). Incluso el trabajo cooperativo, en parejas o pequeños grupos, a propósito de estos one minute papers, es algo cuya utilidad resaltan algunos de los que los han utilizado, haciendo la clase más participativa Graeff, (2010). Otra aportación es la que indica Zeilik (2010), aconsejando una frecuencia de uso regular sin llegar a la saturación, decantándose por una frecuencia semanal o alternativamente, al finalizar un tema o parte del mismo.

La investigación referente a las limitaciones al uso de estas técnicas se centra, según Stead (2005), en la mayor carga de trabajo y en la preferencia de métodos más tradicionales que se consideran más eficaces. Desde el punto de vista de su evaluación, Leeming (2002) argumenta que es preferible no calificar estos breves trabajos (no tiene mucho sentido al menos cuando se trata de que los alumnos den su opinión), pero sí cabe el dar cierto crédito en la calificación, al hecho de haber entregado estos papeles al minuto regularmente, después de todo, es un indicador de asistencia y cuando hay muchas pruebas breves que sí cuentan para nota, no suelen tenerse en cuenta las dos o tres peor hechas. Con esta práctica disminuye la ansiedad y se motiva para hacerlas mejor en otras ocasiones o simplemente para no perder clases.

Estas simples preguntas obligan a los alumnos a reflexionar, haciéndolos sujetos activos de su propia reflexión, de modo que se expresen, para lo cual han de pensar Lerís y Sein-Echalice (2009) obligándolos a sintetizar la información que han aprendido, a relacionar los hechos que ya conocen, o recapacitar sobre su propio proceso de aprendizaje, reflejándolos como métodos low cost, que se han demostrado eficaces para mejorar el aprendizaje de los alumnos.

\footnotetext{
3 Esta técnica insta a los alumnos a que expongan cuál ha sido el punto menos comprendido o el concepto peor entendido de una lección explicada. En realidad, tal cuestión resulta ser un ejercicio interesante y eficaz, puesto que exige que los alumnos, en primer lugar, sopesen y analicen lo que han entendido del tema o asunto expuesto, y, en segundo lugar, que reflexionen y ponderen en breves instantes, la razón o el por qué de tener que seleccionar un concepto $u$ otro como el menos comprendido o el más confuso.
} 
No obstante aunque tiene muy buenos efectos, a un coste muy reducido, Drummond (2007) advierte que hay que saber venderlo a los alumnos

Para ello entiende que el uso de las técnicas del papel al minuto:

a) Pueden tener un cierto peso (por lo general pequeño) en la nota por el mero hecho de haber respondido a estas preguntas, o a una proporción de preguntas previamente anunciada (de alguna manera es también un control de la escolaridad).

b) A veces basta con cambiar el nombre, y en vez de evaluación (que realmente lo es de alguna manera) se puede hablar de una breve actividad de clase, sin connotación alguna con puntos o calificaciones.

Pocos estudios se han realizado en nuestro país difundiendo su utilización, y menos si cabe, valorando los resultados en el campo de la contabilidad. En nuestro entorno podemos mencionar los trabajos de Rivero, Rivero y Gil (2000), Sáez Ocejo y Rodríguez Rodríguez (2004), Sáez Ocejo, Beltrán Varandela y Garrido Valenzuela (2010) y Azkue Irigoyen (2010). Las principales conclusiones de estos trabajos remarcan las anteriores consideraciones y resultados, ya que permiten observar el nivel de satisfacción y utilidad de las técnicas, a pesar de ello se observan pocas diferencias, entre aquellos alumnos usando dichas estrategias de aprendizaje, con respecto a los que no las usaron, observando una mayor utilidad para conceptos teóricos que prácticos, pero con escasa significación.

Parte de la limitaciones de estas metodologías, pueden ser atribuidas entre otras, al poco entendimiento por parte de los estudiantes acerca de su filosofía, las cuales van encaminadas a optimizar la explicación de un tema y la comprensión del mismo en orden al complemento de la técnica de la lección magistral y que a partir de ahí, la labor de estudio y aprendizaje debería ser complementada por otras ayudas, aparte del propio hábito de estudio del alumnado (Sáez Ocejo, Beltrán Varandela y Garrido Valenzuela, (2010)).

En definitiva la valoración final sobre estas técnicas, según Morales Vallejo (2011: 14) es que:

a) Se trata de un método económico, simple y flexible, muy adaptable a todo tipo de materias y situaciones.

b) Los alumnos suelen valorarlos positivamente y son claros los buenos efectos en su aprendizaje en Educación Superior.

c) En conjunto, las ventajas para profesores y alumnos son considerables, si tenemos en cuenta el modesto esfuerzo y tiempo que suponen; una valoración frecuente de estos one minute papers, es que se trata de una de las de las innovaciones didácticas más eficaces para profesores y alumnos (Lerís y SeinEchalice, 2009; Lucas, (2010)).

\subsection{RESÚMENES}

El uso de metodologías más activas implica, necesariamente, el incorporar a nuestro conjunto tradicional de herramientas de trabajo, otras en las que el alumno pase de un aprendizaje memorístico, a un aprendizaje significativo, entendiendo como tal, la adquisición de nuevos conocimientos mediante su inclusión en conceptos ya existentes en la estructura cognitiva del sujeto (Ausubel, 2002). Es en este punto, donde una herramienta tradicional, como es la realización de resúmenes, puede convertirse en una herramienta útil para dar respuesta a algunos de los retos que nos plantea el EEES.

La estrategia de aprendizaje del resumen, es definida como aquella síntesis o abstracción de la información relevante de un discurso oral o escrito, que enfatiza 
conceptos clave, principios, términos y argumentos centrales. Se circunscribe dentro de aquel tipo de técnicas y estrategias de aprendizaje, que permiten dar mayor contexto organizativo a la información nueva que se aprenderá al representarla, en forma gráfica o escrita. Igualmente proporcionan una adecuada organización a la información que se ha de aprender, mejorando su significatividad lógica y en consecuencia, haciendo más probable el aprendizaje significativo de los alumnos, a través de la construcción de "conexiones internas" (Díaz y Hernández, 1999: 5-7). Inmersas en estas estrategias, también podemos incluir las referentes a la representación "visoespacial", como los "mapas conceptuales" 4 o los de representación lingüística, como resúmenes o cuadros sinópticos (Díaz y Hernández, 1999: 3), según se observa en el Cuadro 1.

CUAdRO 1. El RESUMEN COMO ESTRATEGIA DOCENTE EN FUNCIÓN DE SU INCIDENCIA EN EL PROCESO COGNITIVO

\begin{tabular}{|l|l|}
\hline \multicolumn{1}{|c|}{ Proceso cognitivo en el que } & \multicolumn{1}{|c|}{ Tipos de estrategia } \\
\multicolumn{1}{|c|}{ incide la estrategia } & \multicolumn{1}{c|}{ de enseñanza } \\
\hline $\begin{array}{l}\text { Promover una organización más adecuada de la } \\
\text { información que se ha de aprender (mejorar las } \\
\text { conexiones internas) }\end{array}$ & $\begin{array}{l}\text { - Mapas conceptuales } \\
\text { Redes Semánticas } \\
\text { Resúmenes }\end{array}$ \\
\hline
\end{tabular}

La utilización del clásico trabajo-resumen en la docencia universitaria, amplía la capacidad de comunicación entre los que los usan, y amplía la comprensión mutua, entre profesor-alumno, de la docencia y del aprendizaje. Zipitria, Elorriaga y Arruarte (2007:50) argumentan que aquellos estudiantes que resumen de forma eficiente, también guardan información en la memoria de manera más eficiente.

Al igual que los mapas conceptuales, los resúmenes tienen un significado personal, son muy flexibles y de difícil evaluación (cuantificación). Esta limitación es el motivo por el cual, pueden no resultar adecuados en el proceso de evaluación de los alumnos, mediante técnicas cuantitativas. Su utilidad radica en el carácter formativo, puesto que la elaboración de una síntesis por el alumno, evidencia que está aprendiendo significativamente el contenido.

El resumen es algo personal, sobre todo si se pretende obtener provecho de sus ventajas, tales como el incremento de la capacidad de expresión escrita o el desarrollo de ideas siguiendo un hilo conductor. Una de las características de los resúmenes es su consideración como técnica docente activa, no pasiva, de la que se obtiene un mayor provecho particular. La habilidad para sintetizar el contenido de un material, es de gran utilidad para la comprensión y el aprendizaje.

Aunque puedan parecer figuras similares, se observan diferencias entre el trabajosíntesis y el trabajo- resumen, tal y como se muestra en el Cuadro 2 (Noguera 2009: Cap. 15):

\footnotetext{
4 Los resúmenes se diferencian de los "mapas conceptuales", en que éstos últimos son representaciones gráficas con estructuras jerárquicas que muestran cómo se relacionan las proposiciones. Generalmente se utilizan figuras geométricas, como elipses, para encerrar las palabras o enunciados. La similitud de ambas estrategias, radica en que promueven una organización más adecuada de la información, en definitiva, potencian el desarrollo de destrezas, promoviendo el aprendizaje significativo. Sobre su utilidad en la docencia contable española, puede verse con mayor detalle en el trabajo de Canay y Cantorna (2009)
} 
CUADRO 2. DifERENCIAS ENTRE RESUMEN Y SÍNTESIS

\begin{tabular}{|c|c|c|}
\hline CARACTERÍSTICAS & RESUMEN & SINTESIS \\
\hline Qué es & $\begin{array}{l}\text { Reducir la extensión de un } \\
\text { texto copiándolo muy } \\
\text { condensadamente. Implica } \\
\text { reflexionar sobre el texto y } \\
\text { supone generalizar }\end{array}$ & $\begin{array}{l}\text { - Restaura el contenido de un } \\
\text { texto de forma personal } \\
\text { reduciéndolo en términos } \\
\text { breves y precisos según una } \\
\text { redacción personal }\end{array}$ \\
\hline Cómo se hace & $\begin{array}{l}\text { - Se ha de respetar el lenguaje } \\
\text { original, orden y jerarquía del } \\
\text { autor. Ha de ser conciso sin } \\
\text { omisión de puntos relevantes. } \\
\text { - No es necesario usar las } \\
\text { mismas palabras del autor } \\
\text { original, pudiendo usar } \\
\text { sinónimos }\end{array}$ & $\begin{array}{l}\text { - No se necesita respetar la } \\
\text { terminología del autor, ni su } \\
\text { orden. Es más personal que el } \\
\text { resumen }\end{array}$ \\
\hline Fases & $\begin{array}{l}\text { - Lectura comprensiva inicial } \\
\text { - Conexión de ideas claves } \\
\text { agregando nexos para ligar } \\
\text { ideas entre sí } \\
\text { - Establecer un criterio } \\
\text { cronológico, comparativo o } \\
\text { de oposición }\end{array}$ & $\begin{array}{l}\text { - } \quad \text { Lectura comprensiva inicial } \\
\text { - Conexión de ideas claves } \\
\text { agregando nexos para ligar } \\
\text { ideas entre sí } \\
\text { - } \quad \text { Establecer un orden o } \\
\text { jerarquización personal, no } \\
\text { teniendo que ser coincidente } \\
\text { con el autor. }\end{array}$ \\
\hline Evitar & $\begin{array}{l}\text { - Poner calificativos, } \\
\text { comentarios } \\
\text { - Descripción extensa } \\
\text { - No respetar sucesión de } \\
\text { hechos }\end{array}$ & $\begin{array}{l}\text { - Poner calificativos, comentarios } \\
\text { - Descripción extensa } \\
\text { - No respetar sucesión de hechos }\end{array}$ \\
\hline
\end{tabular}

Aunque el modo de elaboración es muy particular, estando en función de las características de cada estudiante, las líneas generales de elaboración de un resumen, son las siguientes: a) Seleccionar la información de la cual se va a elaborar el resumen y b) Subrayar las ideas principales del tema, identificando los conceptos 0 palabras clave del mismo (tales como las palabras técnicas o de mayor uso y relevancia) ${ }^{5}$. La selección de las ideas principales debe seguir tres procesos básicos:

1) Suprimir: todo tipo de ideas triviales o secundarias, al igual que aquellas importantes, pero redundantes.

2) Generalizar: escribiendo varios conceptos o ideas subordinadas (de menor inclusión), en ideas de mayor utilidad (información explícita).

3) Construir y englobar varias ideas subordinadas, en una 0 varias ideas principales (información implícita).

Posteriormente, se recomienda:

- Hacer una lista de las ideas principales.

- Seleccionar el tema central de la síntesis.

- Redactar una versión breve del tema, relacionando las ideas básicas.

- Redactar el resumen según el orden en que aparece la información.

- Finalizar, revisando las ideas principales y las relaciones no establecidas previamente.

En definitiva, tanto desde el punto de vista de la docencia, como del aprendizaje, estas estrategias pueden ser muy útiles en el EEES, ya que respecto a la docencia, permiten mostrar las relaciones entre las ideas principales y las secundarias, de forma

5 Un resumen del proceso puede verse en Marcos Vargas (2009) 
simplificada y estructurada, pudiendo concentrar mucha información en poco espacio. En relación al aprendizaje, potencian la capacidad de concisión, precisión y claridad de un texto; el razonamiento deductivo; las capacidades de análisis, de relación, de orden lógico y de síntesis; la presentación de forma, ordenada y lógica las ideas contenidas en un texto y la comprensión y memorización de la información.

En cuanto a las bondades de la metodología del resumen, sobre la mejora del rendimiento académico, Anaya Nieto (2005: 281-282) argumenta que la actividad de resumir textos leídos, favorece, de forma significativa el rendimiento académico, ya que potencia la comprensión lectora de los sujetos; de hecho diversos trabajos han probado que tienen efectos positivos sobre las habilidades metacomprensivas en diferentes colectivos y situaciones (Rawson, Dunlosky y Thiede, 2000; Rawson y Dunlosky, 2002)

La relevancia, como estrategia de aprendizaje del resumen, es resumida en el Cuadro 3, en el que se detallan los efectos esperados en el alumnado (Díaz y Hernández, 1999, capít. 5):

\section{CuAdro 3. Resumen: Efectos esperados en el ALUMno}

- Facilita el recuerdo y la comprensión de la información relevante del contenido que se ha de aprender, a través de las ideas principales y las relaciones entre las mismas.

- Favorece el recuerdo y el aprendizaje de manera secuenciada y organizada.

- Sirve como modelo para que cuando se aprenda su elaboración, se puedan hacer resúmenes de otros temas o contenidos.

- Permite reconocer, qué conocimientos previos se poseen sobre un nuevo tema, para integrarlos con la nueva información

La extensión ideal de un trabajo-resumen, con respecto al original, debe oscilar aproximadamente entre 1/3 y 1/5 del mismo. Una extensión mayor de 1/3 parte del original carecería de sentido, pues no podría decirse de ella que fuese propiamente un resumen, y ayudaría poco a la hora del repaso. Una extensión menor de 1/5 del original sería demasiado reducida y faltarían en ella datos fundamentales. En muchos libros de texto las ideas están muy esquematizadas, de ahí que resulte difícil hacer un resumen que ocupe menos de la mitad del texto. Las ventajas del resumen son bastante obvias: a) desarrollan la capacidad de síntesis; b) mejoran la expresión escrita; c) facilitan la concentración mientras se estudia; d) simplifican las tareas de repaso y memorización; e) ayudan a ser más ordenado en la exposición y f) perfeccionan la lectura comprensiva.

No obstante, también presentan algunas desventajas, ya que la gran cantidad de tiempo que requieren, aconseja que, aunque sea una técnica útil que debe practicarse, no se use en todos los temas, sobre todo cuando se anda escaso de tiempo. A diferencia del subrayado y del esquema que son útiles y necesarios, el resumen casi siempre es útil, pero casi nunca es necesario, puesto que con el uso de las otras técnicas complementarias, ya se ha producido la necesaria asimilación de los contenidos.

La literatura contable sobre el uso de resúmenes no es muy prolífica. Las principales investigaciones llevadas a cabo en otros campos, ajenos a la disciplina contable, argumentan la capacidad de estas estrategias docentes como ayuda para el alumno, para la comprensión y el aprendizaje significativo (Rawson y Dunlosky, 2002; Anaya Nieto 2005) que ponen en juego numerosos procesos cognitivos implicados en el control de la comprensión de la lectura, y por tanto su potenciación, y en consecuencia, sobre la mejora del rendimiento académico. 
Últimamente se está haciendo hincapié, en una mejora hacia la evaluación de los resúmenes, aspecto bastante complejo, motivado al significado personal que los mismos presentan, ya que son muy flexibles y de difícil cuantificación (Argüelles Álvarez, 2004; Inove, (2005); Zipitria, Elorriaga y Arruarte, 2007), concluyendo que las características principales que nos ayudan a identificar resúmenes inmaduros son: la inclusión de información irrelevante, la copia literal de partes del texto original y más en una época en que existe google) y las dificultades de comprensión. Otro factor a considerar es el nivel de conocimiento del lenguaje o idioma en el que se ha realizado el trabajo. Hasta tal punto esto es así, que el conocimiento lingüístico por sí mismo, puede marcar la diferencia entre un resumen maduro y otro inmaduro.

\subsection{TRABAJOS EN GRUPO}

El "trabajo en grupo o en equipo", es la actividad que se realiza entre más de dos personas y que busca un resultado $u$ objetivo en común. Para ello y siguiendo las técnicas de la dinámica de grupo, son procedimientos sistematizados de organizar y desarrollar la actividad de un grupo de personas. En el campo de la enseñanza, tienen el poder de activar los impulsos y las motivaciones individuales y de estimular tanto la dinámica interna como la externa de los alumnos, de manera que las fuerzas puedan estar mejor integradas y dirigidas hacia las metas del grupo.

En el entorno educativo, los trabajos en grupo adquieren un valor específico de diversión que no sólo estimula emotividad y la creatividad, sino que también introduce dinamismo y tensión positiva en los componentes (Castrillo y Delgado 2010: 1-5). El carácter de este "juego" encierra, además, un doble aspecto: por una parte, implica el hecho de desligarse de la seria situación del momento y, por otra, logra una identificación profunda con los problemas con los cuales se trabaja, con lo cual son ideales para uso complementario a la lección magistral.

En definitiva se pretende que los estudiantes construyan su propio conocimiento, busquen activamente la información, aprendan a formarse y que se autodirigan hacia un proceso de aprendizaje continuado con el objetivo de adquirir aquellas competencias necesarias para su futuro profesional, de modo que les capaciten a desarrollar lo aprendido en cualquier contexto y circunstancia, más allá del entorno universitario y les permita ser competitivos en el mercado laboral, tanto nacional como internacional (Curós i Vila 2009: 2-3).

Las ventajas de los trabajos en grupo, a diferencia de otras técnicas docentes, es que proporcionan un contexto para que, al mismo tiempo, se facilite el aprendizaje en tres terrenos específicos: a) conocimientos y cómo aplicarlos, b) habilidades y c) valores y actitudes. Siguiendo el trabajo desarrollado por los profesores Marín, Millares, García y Vidal (2008: 144-145) algunas de las habilidades destacadas del trabajo en equipo son:

1. Comunicación interpersonal (Christoforou y otros, 2003)

2. Trabajo en equipo (Kalliath y Laiken, 2006)

3. Solución de problemas en grupo (Brewer y Mendelson, 2003)

4. Liderazgo (Jenkins y Lackey, 2005)

5. Negociación (Sheppard, Dominick y Aronson 2004) y,

6. Gestión del tiempo (Jenkins y Lackey, 2005).

El trabajo en equipo, proporciona un aprendizaje más profundo y significativo, siempre y cuando sea usado dentro de un contexto centrado en una metodología activa, ya que demuestra efectos positivos en el rendimiento académico de los alumnos, su motivación y sus actitudes hacia el aprendizaje (Kalliath y Laiken, 2006; Watts, GarcíaCarbonell y Llorens, 2006; Rodríguez Molinuevo, 2010). De hecho los estudiantes las consideran actividades más estimulantes que la docencia tradicional, ya que: 
- Facilitan el desarrollo individual y grupal, en su capacidad de expresión y motivación, generando cambios de actitud, al igual que posibilidades de acción, permitiendo entre los componentes escuchar y ser escuchado, además de la posibilidad de asumir responsabilidades y compromisos con una tarea.

- Respecto a la tarea formativa: potencian la productividad, permiten una mejor generalización del conocimiento, permitiendo ofrecer diferentes puntos de vista, para posteriormente realizar una evaluación grupal y crítica.

Las ventajas del trabajo en grupo son sintetizan en el Cuadro 4:

Como contrapartida, al igual que toda técnica docente, también genera una serie de inconvenientes, ligados al grado de compromiso de cada uno de los componentes, junto con el criterio de organización del trabajo en equipo. Así, en determinados contextos existe resistencia por parte de los estudiantes, ya que no están habituados a esta forma de trabajar (Brooks y Ammons, 2003; Holtham y otros, 2006) por lo que se sienten desorientados o porque consideran que estas actividades les obligan a invertir mucho tiempo (Anson y otros, 2003; Holtham y otros, 2006).

\section{CUAdro 4: Ventajas del tRabajo en EQUipo}

- Se trabaja con menos tensión, al compartir los trabajos más duros y difíciles.

- Se comparte la responsabilidad al buscar soluciones desde diferentes puntos de vista.

- Es más gratificante, por ser partícipe del trabajo bien hecho.

- Se comparten los incentivos y reconocimientos.

- Puede influirse mejor en los demás ante las soluciones individuales que cada persona tenga.

- Se experimenta de forma más positiva, la sensación de un trabajo bien hecho.

- Las decisiones que se toman con la participación de todo el equipo, tienen mayor aceptación que las decisiones tomadas por un solo individuo.

- Se dispone de más información que cualquiera de sus miembros en forma separada.

- Permite distintos puntos de vista a la hora de realizar el trabajo, enriqueciendo el mismo y minimizando las frustraciones.

- Permite el intercambio de opiniones, respetando las ideas de los demás.

- Genera una mayor integración entre las personas permitiendo conocer las aptitudes de sus integrantes.

- Se fortalece el espíritu colectivista y el compromiso.

- Se reducen los tiempos de trabajo, al aportar y discutir en grupo las alternativas.

- Existe un mayor conocimiento e información.

- Surgen nuevas formas de abordar un problema.

- Se comprenden mejor las decisiones, con una mayor aceptación de resultados

Respecto a la evaluación para este tipo de fórmulas, también se suelen generar problemas ya que, puede ser necesario asignar una nota individual a los estudiantes, aunque el trabajo lo hayan hecho en grupo, debido a la aparición de comportamientos oportunistas entre los componentes (Brooks y Ammons, 2003; Holtham y otros, 2006 y Michaelson, 2003). Ello conlleva a que los docentes, tal y como mencionan Marín, Millares, García y Vidal (2008: 146-147) deban implicarse e invertir mayor tiempo al objeto de motivar a los alumnos, supervisando actividades y el proceso del grupo, que no siempre es fácil de evaluar.

Otra de los obstáculos de esta metodología, sobre todo en los primeros cursos, surge de la poca preparación de los alumnos para este tipo de cooperaciones en equipo, debiendo de aprender a trabajar con otros compañeros, por lo que precisan tiempo, formación y práctica (Young y Henquinet, 2000). El Cuadro 5, incluye una síntesis de los principales inconvenientes: 


\section{CUADRO 5: INCONVENIENTES DEL TRABAJO EN EQUIPO}

- Grado de compromiso de cada uno de los componentes

- Criterios de organización del trabajo en equipo

- Resistencia por parte de los estudiantes, por la falta de costumbre al trabajo en equipo

- Gran inversión de tiempo en el trabajo

- Aparición de comportamientos oportunistas

- Grado de implicación e inversión de tiempo y supervisión del profesor

- Gran inversión inicial en la formación y preparación del alumnado para la preparación del trabajo en grupo en los primeros cursos

Esas ventajas e inconvenientes antes citados, siguen llevando a los investigadores a valorar sobre la mejor manera de articular, gestionar y evaluar esta estrategia docente. De lo que no cabe duda, es que el papel del profesor cuando se introducen grupos en el aula, independiente del tipo de metodología aplicada (tradicional $o$ activa), sólo se obtienen ventajas, como las señaladas cuando los docentes diseñan, guían el proceso y lo evalúan adecuadamente (Oldham y otros, 2006; Callialta y Lavquen, 2006).

Para Arquero y otros (2001), dentro del EEES, el alumno debe desarrollar diversas competencias transversales, entre las que ha de destacar, la capacidad de trabajo en grupo, al objeto de obtener un adecuado desempeño profesional futuro, para lo cual se necesitan nuevas metodologías que ayuden a conseguir dichos objetivos, no quedándose sólo en el resultado final del trabajo entregado (entrega del trabajo final y exposición del mismo en público), también se debe de evaluar el proceso de trabajo en grupo, evaluando sus actividades (Gómez Ruíz y Naranjo-Gil (2010:18), lo que nos adentra dentro de otras líneas de investigación centradas en el aprendizaje por trabajo cooperativo'.

Los estudios sobre trabajo en equipo, nos indican que estos grupos se pueden emplear en cualquier disciplina universitaria, bien como complementarias a la lección magistral (dentro de una docencia tradicional) o bien como nuevas metodologías, como el "aprendizaje basado en el trabajo" o el "aprendizaje autónomo" (Michael son, 2003). Aún así, el papel del profesor no termina con el diseño de la actividad y la formación de los grupos, siendo necesario un proceso de sensibilización previa para su preparación de cara al trabajo futuro de modo efectivo, lo que puede consumir un excesivo tiempo a la explicación de contenidos (Young y Enquiñe, 2000).

Por otra parte, es recomendable dar a los alumnos una buena descripción de qué es exactamente lo que el profesor quiere lograr con la actividad de grupo (Sheppard y otros, 2004), pero siendo también importante comunicar, cómo se les sugiere que trabajen y sobre todo, cómo se evaluará el proceso del grupo, detallándose lo máximo posible. Evidentemente todo el proceso va a implicar una mayor dedicación a la supervisión por parte del profesor (Callialta y Lauquen, 2006).

El trabajo del grupo se compone de dos partes que no siempre son fáciles de diferenciar por los alumnos o los profesores: producto y proceso 7 . El proceso del grupo afecta tanto a la calidad del producto final como a la motivación de los estudiantes. Sin embargo, no es fácil encontrar criterios específicos que nos permitan identificar

\footnotetext{
6 La diferencia entre trabajo en grupo y trabajo cooperativo, es diferenciada por Castrillo Lara y Delgado Hurtado (2010:3-6) en la que básicamente se establecen dos condiciones para que un grupo cooperativo tenga éxito: establecer objetivos de grupo y aportación individual, y entre los elementos necesarios para una correcta implementación, se necesita: una interdependencia positiva y una responsabilidad individual.

7 Producto: lo que el grupo tiene que presentar (informes, propuestas, presentaciones orales) y proceso: el modo en que el grupo desarrolla sus actividades y por otra parte, los comportamientos de los componentes del grupo (Marín, Millares, García y Vidal, 2008: 148).
} 
conductas observables como buenos procesos de grupo. La literatura del estado de la cuestión, resume esta serie de criterios relevantes, todos ellos muy relacionados (Marín, Millares, García y Vidal (2008: 148).

- Frecuencia de participación y asistencia a reuniones (Dance y Kamvounias, 2005)

- Calidad de las intervenciones dentro del grupo o de los documentos entregados (Brooks y Ammons, 2003)

- Preparación de las reuniones y procesamiento de la información previa a la reunión (Sheppard y otros, 2004)

- Comunicación entre miembros del grupo (Brooks y Ammons, 2003)

- Delegación sin dominación (Rugarcia y otros, 2000)

- Aceptación de responsabilidades (Michaelson, 2003)

- Manejo adecuado de los conflictos y toma de decisiones en equipo (Sheppard y otros, 2004)

De todos ellos, uno de los que más preocupa, tanto a profesores como alumnos, es el que hace relación al comportamiento oportunista de algunas personas del grupo, como por ejemplo, no cumplir con la función individual designada, abandono, actitud pasiva o no asistencia a reuniones previstas, generando descontento en el grupo, situación que se produce cuando se trabaja con más de cuatro personas o cuando el grupo trabaja junto, fuera de las hora de clase (Brooks y Ammons, 2003; Kalliath y Laiken, 2006; Willcoxson, 2006). Una de las formas de evitar ese comportamiento parásito de los alumnos, es que los grupos establezcan normas de funcionamiento (Sheppard y otros, 2004).

Otro de los procesos al que se le debe prestar atención, es el referente a la evaluación de este tipo de estrategias, en los que en la docencia tradicional sólo se suele evaluar el producto final; no obstante, cuando se introducen metodologías activas para formar a los alumnos, también en competencias transversales, es preciso incorporar el proceso del grupo a la evaluación de la asignatura, con el objeto de hacer visible al alumnado, la relevancia del modo en que se han conseguido los productos (Dancer y Kamvounias, 2005).

La literatura en este sentido es coincidente en indicar que la evaluación del proceso, puede utilizarse ofreciendo feedbacks a los alumnos, sobre cómo están actuando y qué cosas podrían mejorar, recomendando evaluaciones periódicas y con múltiples ítems de evaluación, en vez de sólo una evaluación final del curso (Young y Henquinet, 2000). A pesar de ese consenso existente, aparecen opiniones en contra cuando se plantea que la nota del proceso de grupo afecte, en determinado porcentaje, a la nota final de la asignatura, fundamentado en la dificultad para establecer criterios de evaluación objetivos, sobre todo si los grupos no trabajan en clase (Brooks y Ammons, 2003; Willcoxson, 2006).

El evaluar entregando una misma nota a todos los miembros del equipo, o discriminar la nota en función de las contribuciones individuales al proceso del grupo, siempre ha sido motivo de discusión (Lloret y Marin-Garcia, 2007), y las investigaciones publicadas, no resuelven esa duda, pero coinciden en afirmar que evaluar adecuadamente el proceso del grupo, ayuda a los alumnos a centrarse en el modo en que trabajan como equipo, constituyendo por si sólo, una experiencia de aprendizaje (Watts y otros, 2006). Para ello, aparte de los comentarios previamente apuntados, se han utilizado diversos métodos como las parrillas de evaluación en base a determinados criterios (siendo el docente evaluador) y comentarios escritos del alumnado sobre el funcionamiento del grupo (usando a los alumnos como autoevaluadores de su labor o de sus compañeros (Christoforou y otros, 2003; Watts y otros. 2006).

$$
\text { educade, no 2, 2011, p. } 14
$$


En España algunas de las últimas aportaciones, sin ánimo de ser exhaustivos, realizadas sobre experiencias docentes de trabajo en grupo en el campo de la Contabilidad [Carrasco y otros (2009), Rodríguez Molinuevo (2010), Baraldés Capdevila (2010) y Gómez Ruíz y Naranjo-Gil (2010), Aldazábal y Espinosa (2011)] 8, coinciden en el alto grado de satisfacción con el uso de estas estrategias, observando una mejora tanto de atención, implicación y calificación del trabajo de los alumnos a nivel grupal, aunque no refrendado por los resultados a nivel individual, en el que se puede apreciar que los estudiantes trabajan bien a nivel de grupo, pero por el contrario, individualmente no alcanzan el mínimo exigido de conocimientos. Una de las explicaciones argumentada por Baraldés Capdevila (2010: 5) se argumenta en el poco tiempo de estudio individual que invierten los alumnos, es decir la falta planificación, dedicación y continuidad de trabajo individual fuera de clase.

\section{EXPERIENCIA DOCENTE EN LA ESCUELA UNIVERSITARIA DE ESTUDIOS EMPRESARIALES DE VIGO}

\subsection{CONTEXTO DEL ESTUdIO, LA ASIGNATURA, tÉCNICAS DOCENTES APLICADAS E HIPÓTESIS DE tRABAJO.}

Con el objeto de analizar y valorar estas técnicas docentes, se realizó un estudio empírico de las mismas, en un curso de la Diplomatura en CC. Empresariales de la Escuela Universitaria de Estudios Empresariales de Vigo, en la asignatura "Contabilidad Financiera l", perteneciente al $1^{\circ}$ año, materia de 12 créditos, hoy ya extinta del citado plan de estudios.

Las estrategias docentes empleadas fueron las mencionadas previamente en el segundo apartado, es decir: a) las fórmulas de "papel al minuto", b) la realización de trabajos individuales tipo-resumen y c) el trabajo en grupo.

Respecto a la primera de ellas, técnicas del "papel al minuto", el procedimiento para su aplicación, se circunscribió a estas características:

Los primeros días de clase, se explicaron los objetivos y motivaciones perseguidas, al igual que el proceso, tiempos y frecuencias de empleo durante el curso. Así, al finalizar una sesión teórica, se les pedía a los alumnos que rellenaran un papel indicando: a) los conceptos que menos habían entendido de la explicación previa, y b) la contestación a dos preguntas cortas, formuladas por el profesor, sobre el tema impartido previamente. Después de un tiempo de reflexión (de unos tres minutos), los alumnos contestaban las respuestas en un pequeño papel-nota. Finalizada la fase de selección y clasificación, en la siguiente clase, se comentaban y contestaban a los estudiantes, de un modo general, los aspectos más dudosos planteados, al igual que la contestación de las preguntas formuladas por el docente, analizando los errores cometidos, haciendo una valoración generalista de los mismos, con el fin de puntualizar aquellos fallos cometidos y profundizar en los conceptos. Con el fin de motivar a los alumnos, se les indicó que las preguntas formuladas por el educador, serían utilizadas en el examen de teoría de junio, al objeto de valorar la técnica en cuestión?.

\footnotetext{
8 Relacionado con el tema, aparte de los trabajos citados, recomendamos al lector el resumen de Castrillo y Delgado (2010) sobre el aprendizaje cooperativo, que recoge los principales resultados empíricos obtenidos sobre la materia en cuestión y más en concreto sobre: mejoras de resultados y actitud del alumno, así como sus condicionantes: formación de grupos y criterios de evaluación.

9 Para dicha prueba, se incluyeron un total de 7 cuestiones; de este total, 4 fueron elegidas entre todas las preguntas formuladas por el docente, de las incluidas en una de las estrategias del papel al minuto (en concreto la referente a las dos cuestiones planteadas al final de las sesiones teóricas).
} 
Para la segunda metodología, realización de "trabajos-resúmenes", se propuso la realización de una síntesis, de una a dos páginas, por cada tema perteneciente al texto básico seguido en la materia, los cuales eran entregados a medida que se finalizaba cada parte del programa, dejando entre una a dos semanas para su elaboración. El docente no penalizó el incumplimiento de la obligación. Igualmente tampoco se les comunicó, que el día del examen final de junio, se permitiría a aquellos alumnos que entregaran en tiempo y forma dichos trabajos, su uso en la parte práctica de la prueba. Por otra parte, y como política de motivación, también se permitió, con el conocimiento previo por el alumnado, antes de la prueba final, la posibilidad de hacer el resumen de una lección del temario de Contabilidad Financiera, y contar con el mismo en la parte teórica, a cambio de que una de las preguntas realizadas en la prueba final, perteneciera a dicho tema resumido.

Con respecto a la tercera estrategia docente, la realización de un "trabajo en equipo", se repartió la clase en grupos de tres personas máximo (participando en la experiencia 11 grupos), encomendando a los alumnos la realización de un trabajo, consistente en la preparación de una clase magistral sobre un tema de la asignatura, el cual sería defendido posteriormente en el aula. La elección del grupo que impartiría la sesión magistral se realizaría bajo sorteo, dejando al resto la posibilidad de formular aquellas preguntas oportunas al grupo expositor. A su vez, en el examen final, una de las preguntas del examen práctico, consistió en la resolución de un problema, sobre el tema preparado por los grupos, al objeto de verificar el nivel alcanzado con la estrategia docente, aspecto del que no se informó al alumnado.

Los objetivos de la experiencia, como hipótesis de trabajo y de acuerdo a la literatura previa comentada en la sección anterior, fueron los siguientes:

1. Valorar el grado de satisfacción y aceptación, además de su utilidad y motivación, de estas metodologías para el alumnado.

2. Comprobar, si estas metodologías podían incrementar los conocimientos y resultados académicos de los alumnos, mejorando por tanto, sus competencias.

Para poder llevar a cabo la valoración de esta experiencia, resulta imprescindible conocer las impresiones de los alumnos, así como analizar los resultados obtenidos en sus calificaciones. Con ese fin, se elaboraron dos cuestionarios (ver anexo I y anexo II), ambos de carácter anónimo. El primero de ellos, fue presentado a los alumnos asistentes, al finalizar la docencia de la asignatura, durante los últimos días de clase, y el segundo cuestionario, inmediatamente después de la realización de la prueba final de junio. En el apartado de resultados analizamos, no sólo los estadísticos descriptivos, sino también la aplicación estadística sobre los datos del censo de alumnos, para ratificar si las diferencias entre porcentajes de alumnos que aprobaron la materia en el examen de junio, en orden al uso de las técnicas docentes señaladas, eran significativas o no.

\section{ANÁLISIS DE RESULTADOS}

\subsection{Aspectos Generales Relacionados CON la EXPERIENCIA: APRECIACIÓn DEL ALUMNADO}

Las respuestas emitidas por los alumnos en los cuestionarios permiten concluir la relevancia y nivel de dificultad que le conceden a la asignatura de "Contabilidad Financiera I", considerando que se debe estudiar muy frecuentemente (ver anexo 0 , apartados Dy H). La asistencia diaria fue descendiendo a medida que transcurría el curso, aún a pesar de que los estudiantes manifestaron que acudieron con bastante regularidad, como se aprecia en el Gráfico 1. 
Gráfico 1. Asistencia a Clase (Número de estudiantes)

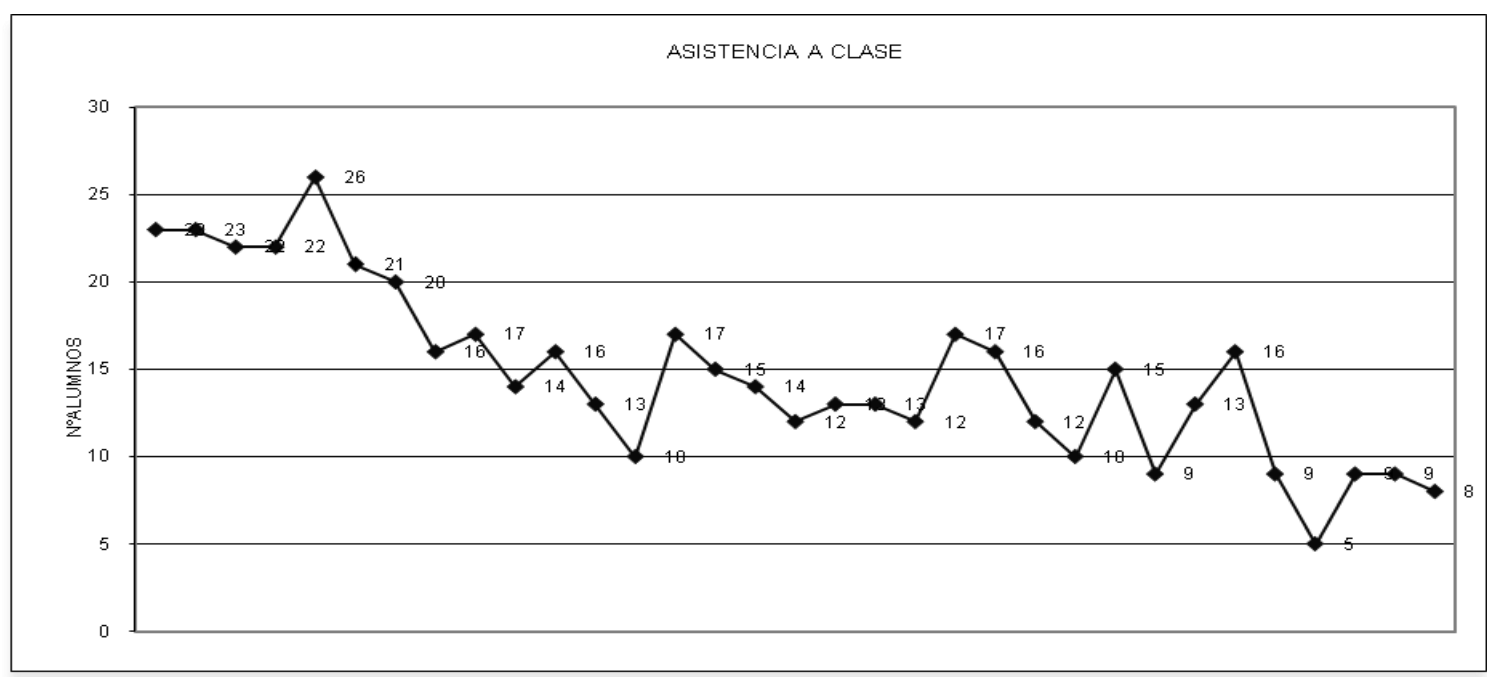

Gráfico 2. Utilidad de LAS estrategias del papel al MinUto
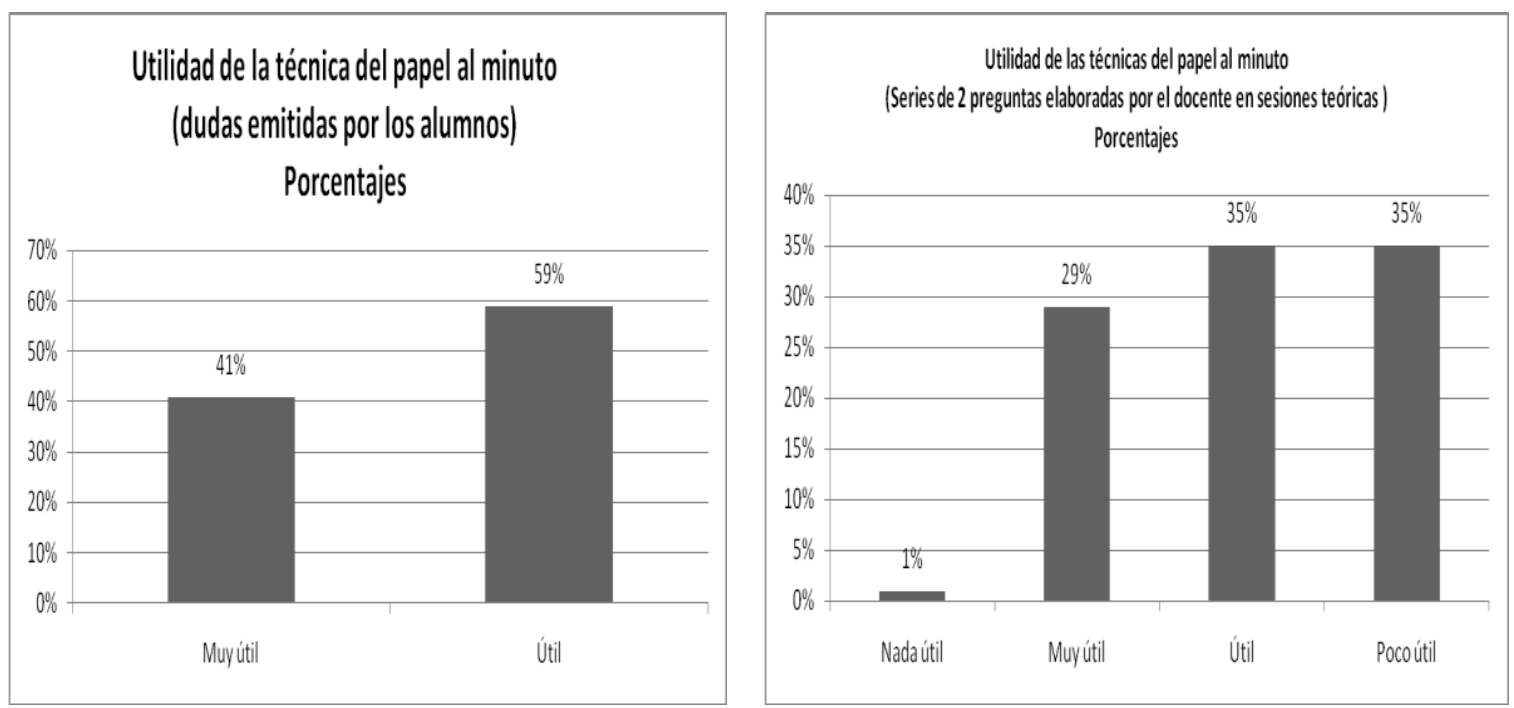

El nivel de utilidad y satisfacción del alumnado con el uso de estas metodologías ha sido muy alentador, ya que todas las actividades propuestas se consideraron muy útiles por un amplio abanico de estudiantes. De todas ellas, las más valoradas por los alumnos ha sido: una técnica del papel al minuto (referente a la indicación de dudas surgidas después de la lección magistral, en las que el 100\% consideró "útil" o "muy útil" la propuesta empleada, tal y como se observa en la parte izquierda del Gráfico 7) y la estrategia del trabajo en grupo (en la que un $94 \%$ de los estudiantes consideró que habían aprendido más que suficiente con la experiencia una vez realizada la misma, ver anexo 0, apartado F). Respecto a la otra técnica del papel al minuto, el $64 \%$ constató la alta idoneidad de las cuestiones planteadas por el profesor como ayuda para el proceso de aprendizaje (ver parte derecha del Gráfico 2), al igual que con respecto a la comunicación y posterior explicación de las dudas suscitadas, refrendado por el apoyo del alumnado por un $90 \%$ de respuestas que así lo confirmaban ${ }^{10}$.

10 Sólo un 1\% consideró "nada útil" esta estrategia docente. La percepción de los alumnos ante la actitud de los docentes, a la hora de contestar las cuestiones dudosas y a las 2 preguntas 
El resto de metodologías, aunque con menor grado de satisfacción, también cumplen como estrategias a tener en cuenta, por parte del alumnado, los cuales valoran su beneficio cara al aprendizaje de conocimientos, como se observa en el Gráfico 3, en el que se aprecia que un amplio porcentaje de estudiantes valora positivamente el trabajo grupal (67\%) y el aprendizaje a través de resúmenes (70\%). Cabe destacar, respecto a esta última, que la apreciación inicial fue muy similar, tanto antes como después de realizar la prueba final de junio, si nos atenemos al nivel de aprovechamiento del aprendizaje, ya que un $7 \%$ y un $56 \%$ apuntó que "siempre" o "casi siempre", respectivamente, se había aprovechado, al menos inicialmente, de la técnica en cuestión para consolidar sus conocimientos sobre la materia (ver anexo 0 , apartado I). En este sentido, la creencia de haber elaborado unos buenos documentos síntesis se manifiesta por igual, tanto antes, como después de hacer la prueba final de junio, con resultados parejos de un $63 \%$ y $66 \%$ respectivamente (ver anexo 0 , apartados $K$ y $M$ )

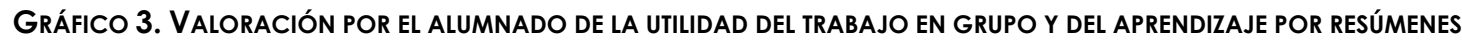
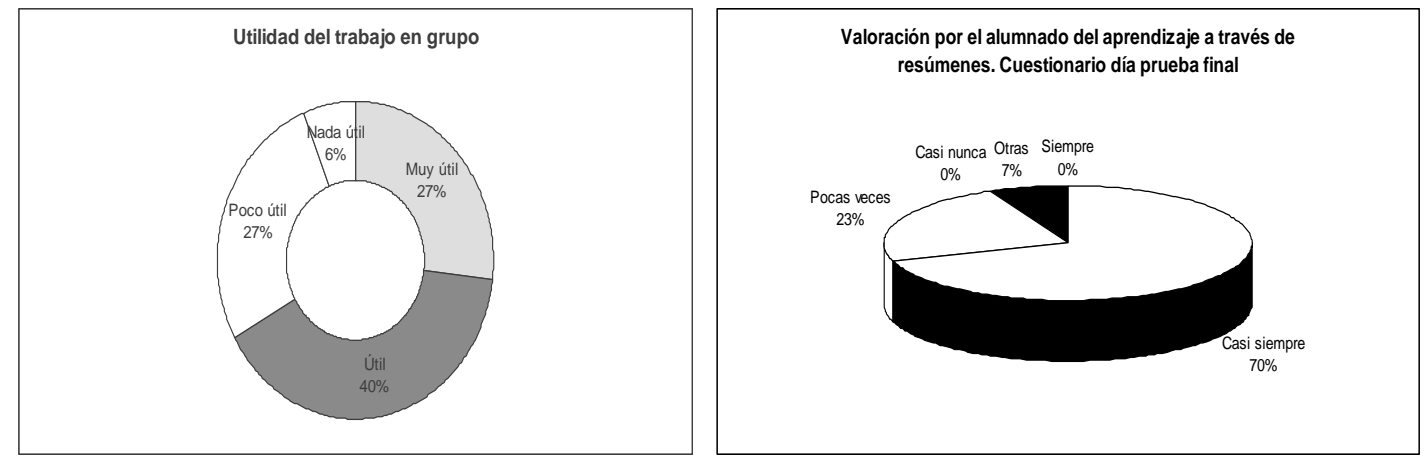

Por lo que respecta a la frecuencia de uso de estas metodologías, como viene siendo tónica, no es muy elevada, ya que en ningún caso son utilizadas por más de la mitad del alumnado, salvo el caso de los trabajos individuales a través de resúmenes, que son usados habitualmente por más del $53 \%$ de los alumnos. La asistencia a las aulas, es un hecho poco común en los últimos tiempos, aunque con el nuevo sistema del EEES esperamos se soslaye este hecho, ello confirma la relación existente entre el poco uso de estas fórmulas por parte del alumnado y la falta de alumnos en la universidad, pudiéndose constatar en las estrategias dos fórmulas usadas del "papel al minuto", en las que sólo un $47 \%$ de la muestra, participó con frecuencia en la contestación de las dos preguntas realizadas por el docente, por un $27 \%$, con respecto a la otra metodología utilizada, es decir, la referente a la indicación de dudas surgidas en la sesión magistral.

La realización del trabajo grupal también confirma la tendencia general, en la que se constató la participación con carácter asiduo, de sólo un $36 \%$ de los encuestados ${ }^{11}$. Como hecho destacable resulta significativo comprobar que, aproximadamente más

formuladas fue positiva, realizando: "siempre" o "casi siempre" en un $70 \%$ y $30 \%$ respectivamente, su labor.

11 En un principio se formaron un total de 11 grupos divididos entre 2 a 3 componentes. Del total de grupos, el $27 \%$ no se presentó el día de la exposición (el total de todos los miembros), mientras que de los grupos restantes, dejaron "colgados" a sus compañeros un $45 \%$ (el $17 \%$ de los alumnos que inicialmente se comprometieron a realizar el trabajo). Por tanto, se pudo constatar una tasa de abandono del $38 \%$ de los estudiantes, que inicialmente habían valorado la posibilidad de hacer la tarea en equipo. 
de la mitad de la clase, apenas participó activamente en las metodologías previstas por los docentes (ver anexo 0, apartados A, B, C y G).

\subsection{CRíticAs negativas a las tÉCNICAS DOCENTES y SOlUCIONES PROPUESTAS POR PARTE DEL ALUMNADO}

Aunque en líneas generales la actitud de los alumnos ha sido muy positiva, muchos de ellos han rechazado de plano la impartición de estas nuevas metodologías, aún a pesar de que reconocen su apoyo como ayuda para la comprensión de la materia y la aplicación correcta por parte de los docentes.

Así, aquellos alumnos que manifestaron el "poco" o "nulo" provecho a las fórmulas del "papel al minuto", justificaron sus respuestas argumentando que en el que caso que tuvieran dudas, se las harían saber al profesor y que la realización de las dos preguntas al final de la clase teórica, no las consideraban pertinentes, ya que en ese momento se "estaba saturado de información y era muy difícil sintetizar todo lo aprendido", pudiendo ser más interesante el "realizar las preguntas en la sesiones siguientes", razonamiento comprensible, aún a pesar de que el objetivo de las mismas era el de ayudarles a comprender la materia a través de dichas estrategias, situación a priori no entendida.

Otra de las observaciones de la experiencia, fue la relativa al poco nivel de detalle y a la falta de profundidad respecto a las dudas emitidas, que llevaba a los estudiantes a formular cuestiones muy vagas y poco concretas. Ello puede atribuirse a la falta de conocimientos previos de la materia y a que el nivel de los alumnos todavía es bajo, aparte de la falta de vocabulario técnico, propio de los alumnos de los primeros cursos.

El trabajo de Sáez y Rodríguez (2004: 20-21) confirma el hecho de que los estudiantes, no suelen entender la filosofía de las herramientas del papel al minuto, las cuales van encaminadas a optimizar la explicación de un tema y la comprensión del mismo como complemento de la lección magistral y que a partir de ahí, la labor de estudio y aprendizaje debería ser complementada por otras técnicas docentes.

Por lo que respecta al trabajo individual a través de resúmenes, la mayor fuente de críticas se fundamenta en la escasa relación coste-beneficio derivada del esfuerzo de su uso, debido a la gran carga de trabajo que conlleva su elaboración, realizando los mismos únicamente con el objetivo de cumplir con las exigencias del docentel2-, aparte de la anterior, el resto son resumidas en el Cuadro 6.

\section{CUADRO 6. MOtIVOS DE ESCASA UTILIDAD DE LOS RESÚMENES COMO TÉCNICA DOCENTE}

- Mayor relevancia de otros métodos de estudio, en detrimento de los resúmenes

- Escasa relación coste-beneficio medida en tiempo de aprovechamiento

- Elaboraron de los trabajos con el objetivo de cumplir con las exigencias del profesor y no con el fin de sintetizar conceptos, cara al aprendizaje de la materia.

- Elaboración con el objetivo de cumplir con las exigencias del docente, sobre todo si el tiempo para su elaboración era escaso.

- Se considera mayor utilidad para el aprendizaje teórico que para el práctico.

En cuanto a la dinámica de grupos, los principales motivos argumentados para mencionar su escasa utilidad se sintetizan en el Cuadro 7.

\footnotetext{
12 De hecho fueron consideradas "poco" o "nada" útiles por un $30 \%$ de los encuestados (ver anexo 0 , apartado E)
} 


\section{Cuadro 7. Críticas de los alumnos a los trabajos en Grupo como estrategia docente}

- Elección de compañeros de equipo

- Falta de organización entre equipos

- Excesiva pérdida de tiempo malgastada en la organización (tiempo en reuniones, falta de organización, calendario de reuniones)

- Preferencia de elaboración del trabajo individualmente.

- Exposición del trabajo (por librarse de la exposición y por la no exposición por todos los grupos)

Un factor muy relevante fue la elección de compañeros de equipo, ya que se pudo considerar por sus argumentaciones, la falta de organización existente entre algunos de los equipos, refrendada por la excesiva pérdida de tiempo malgastada en dicha organización (tiempo en reuniones, falta de organización, calendario de reuniones) ${ }^{13}$ que en un $33 \%$ de los encuestados, aconsejaba la preferencia de elaboración del trabajo individualmente, tal y como se puede apreciar en el Gráfico 4.

\section{Gráfico 4. Preferencia del trabajo individual VS. trabajo en Grupo}

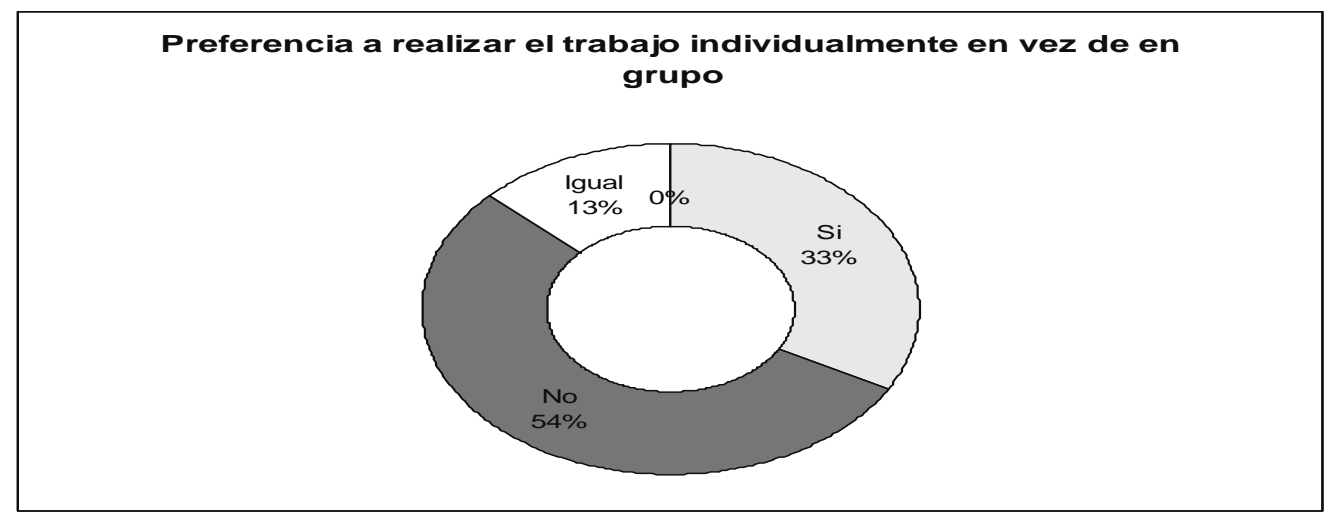

Otra de las críticas se formuló en relación a la exposición del trabajo, ya que al ser un amplio número de equipos, le llevó al equipo docente a tomar la decisión de que la misma fuera realizada sólo por un grupo, y no por todos, lo que generó cierta frustración en aquellos grupos de alumnos que trabajaron duramente, y no impartieron oralmente la lección magistral del tema, y por el lado contrario, los que tuvieron la suerte de no exponer el trabajo, por el miedo escénico que ello conllevaba, con lo cual se libraron de hacerlo. Ante la sugerencia de valorar la preferencia de la impartición de los temas realizados por el propio docente, en vez de mediante la elaboración del trabajo y posterior exposición por parte del grupo, sólo el $27 \%$ hubiera preferido que el docente utilizase la lección magistral, mientras que un $40 \%$ fue partidario de la estrategia utilizada (ver anexo 0 , apartado J).

Las sugerencias aportadas por los alumnos, al objeto de mejorar la potencialidad de estas metodologías docentes, vienen del lado de la evaluación; en definitiva, les prima con más relevancia, el aspecto formal relacionado con la superación de la asignatura, que el objetivo de aprendizaje. En ese sentido, argumentan que podría ser positivo, obtener una contraprestación en la calificación final de modo que se motivase a los mismos.

Igualmente y para una de las técnicas del papel al minuto (realización de dos preguntas) también se sugirió, que se premiaran aquellas contestaciones correctas,

13 De hecho, un $20 \%$ de los alumnos de la muestra, manifestó haber tenido problemas en el equipo con otros compañeros. 
elevando la nota en la calificación final, es decir, valorando dichas metodología como un pequeño control, que en caso de superarse, debería implicar un incremento de calificación. La misma línea argumental fue utilizada para el trabajo-resumen. Punto y aparte es la evaluación del trabajo grupal, ya que implicaría una mayor coordinación y seguimiento por parte del docente, no sólo valorando el producto, sino también el proceso, lo que haría necesario calificar de algún modo, el nivel de compromiso y trabajo en equipo, al objeto de homogeneizar la calificación entre todos los componentes del grupo, con el fin de evitar conflictos de intereses, penalizando negativamente, todo tipo de abandono para aquellos estudiantes que incurrieran en dejación de sus funciones, contribuyendo con ello a mejorar las competencias transversales que los nuevos grados exigen. No olvidemos que la estrategia del trabajo en equipo, generó un alto nivel de abandono entre los estudiantes, que tuvo como consecuencia, una cierta frustración entre el resto de componentes.

Otra forma de consolidar la técnica docente grupal, partía del hecho de que los alumnos podrían exponer algunos temas, bien al profesor, bien a sus compañeros (con esta última, las sinergias podrían incrementarse), por un lado, al objeto de evitar la frustración de no poder demostrar sus conocimientos en público, después de todo el trabajo realizado, y por otra parte, para calificarles por ello, al objeto de motivar a todos los grupos.

A nivel más general y como cuestión abierta, se les sugirió a los alumnos que comentaran, de un modo más explícito, algunas posibilidades de mejora para estas estrategias docentes, al objeto de optimizar su uso; a pesar de ello, las propuestas apuntadas se centraron más en cuestiones generales de aprendizaje de la asignatura, que en la mejora de las técnicas docentes propiamente impartidas, las cuales hemos resumido en el Cuadro 8.

Como se puede observar, aparte de las incluidas en otras cuestiones de la encuesta, las principales sugerencias se remitieron a la posibilidad de realizar más ejercicios en clase de carácter práctico, aspecto lógico si tenemos en cuenta que es una asignatura en la que prima fundamentalmente tal hecho. Otra de las recomendaciones hizo referencia, a la exigencia de una evaluación continua de los trabajos o labores realizadas, ya que el mero hecho de aprender, no es suficiente para un amplio colectivo y quieren que su esfuerzo tenga recompensa. Esta actitud es coherente, nadie trabaja gratis, y el binomio esfuerzo-evaluación debe ser directamente proporcional. Otro aspecto que llama la atención es la conveniencia de hacer controles parciales de la asignatura, justificada con el mantenimiento de cierta tensión, por parte del docente hacia los estudiantes, lo que ya les obligaría a estudiar con mayor asiduidad por motivos obvios.

\section{CuAdro 8. Propuestas de MEJORAS Y SUGERENCIAS DE APRENDIZAJE DE LAS ESTRATEGIAS DOCENTES}

- Realización de más ejercicios prácticos en clase, alternados con la teoría (12 sugerencias)

- Valoración de los trabajos o labores realizadas mediante una evaluación continua para incentivar al alumno (4 sugerencias)

- Hacer más ejercicios a diario, llevándolos a casa y corregirlos en la/s clase/s posterior/es (2 sugerencias)

- Existencia de más tensión en clase para un mayor aprovechamiento (1 sugerencia)

- Realización de controles parciales durante el curso, aunque no eliminen materia, al objeto de obligar al estudiante a un aprendizaje más continuo de la asignatura (1 sugerencia)

- Realización de ejercicios en el encerado, no en transparencias (1 sugerencia)

- Realización de exposiciones semanalmente (1 sugerencia)

- Docencia de menos a más, es decir incluir los conceptos poco a poco, no empezando con vocabulario complejo (1 sugerencia)

- Realización de otros métodos, aparte de los utilizados, para motivar el trabajo en equipo e individual, al objeto que la clase fuese más amena (1 sugerencia) 
Un hecho que podría ser objeto de debate es el referente a la preferencia de mantener la estrategia de aprendizaje habitualmente usada en el Bachillerato o en ciclos superiores de Formación Profesional, en referencia a la continuidad de esfuerzo y evaluación continua de la materia, en la que prima con mayor profusión el trabajo diario, con su correspondiente evaluación, más que el procedimiento habitual hasta la fecha utilizado en la Universidad, hasta ahora llamémosla tradicional, fuera del entorno de Bolonia, en la que se transfiere la evaluación del esfuerzo de aprendizaje, a la realización de sólo un examen final. En otras palabras, los estudiantes demandan mayor control por parte de los profesores, tanto para su aprendizaje como para su evaluación, en eso parecen coincidir con la filosofía que emana del EEES. Como hecho anecdótico, a algunos alumnos, no les gusta la resolución de ejercicios a través de transparencias, sino aquellos realizados en el encerado con tiza, entendemos porque el uso del mismo les permite un mayor grado de reflexión y maduración de los conocimientos adquiridos.

\subsection{VALORACIÓN DE LOS RESULTADOS OBTENIDOS}

La segunda de las hipótesis a tener en cuenta, en orden a comprobar, si estas metodologías podían incrementar las competencias en conocimientos, vía resultados académicos, permitieron apreciar, globalmente pocas diferencias entre aquellos alumnos que habían superado la asignatura usando todas las estrategias de aprendizaje (30\%) que respecto a los que no las usaron (27\%). En el apartado teórico se comprobó un porcentaje de aprobados (40\%) sensiblemente mayor, para aquellos alumnos que utilizaron las estrategias docentes, que respecto a aquellos que no las utilizaron (33\%).

Lo que también parece corroborarse es que aquellos alumnos que superan la parte teórica, también suelen superar la parte práctica, independientemente del uso de las estrategias usadas. Los Gráficos 5 y 6 permiten observar las calificaciones obtenidas por los estudiantes que, tanto usaron como no, las técnicas docentes señaladas, distinguiendo entre parte teórica y práctica.

Gráfico 5.CALIfICACIONES de ALUMNOS QUE USARON LAS ESTRATEGIAS dOCENTES

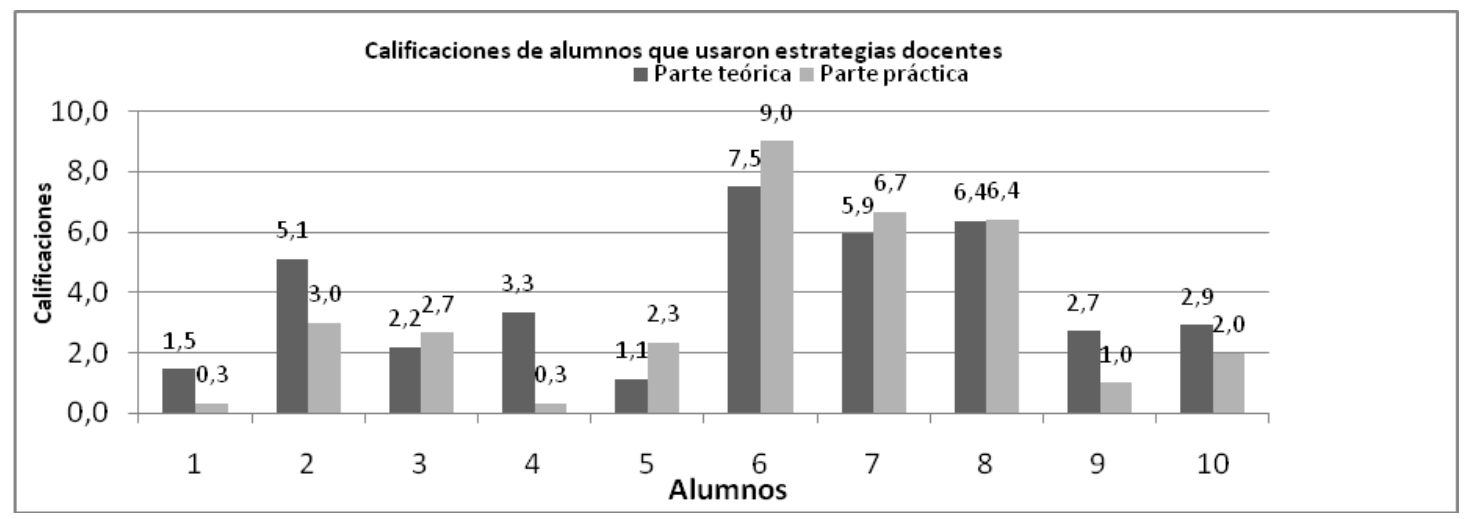

En el apartado práctico, esta tendencia también se mantiene, aunque con menor fuerza ya que, se comprueba un índice de aprobados del $30 \%$ para aquellos alumnos que habían participado en el uso de las técnicas, por un $27 \%$ de estudiantes que nos los utilizaron. A nivel particular estos resultados mantienen la tónica general, antes mencionada, no sobresaliendo ninguna metodología con respecto a las otras. En concreto, con la primera de las fórmulas del papel al minuto empleada (realización de dos preguntas al final de cada sesión de teoría), se observaron puntuaciones más negativas que positivas en el examen de junio. En ninguna de las preguntas 
formuladas, se obtienen porcentajes medios por encima del aprobado (valor 1), tal y como se puede apreciar en las estadísticas del Cuadro 914.

Gráfico 6. Calificaciones de alumnos que no usaron LAS estrategias docentes

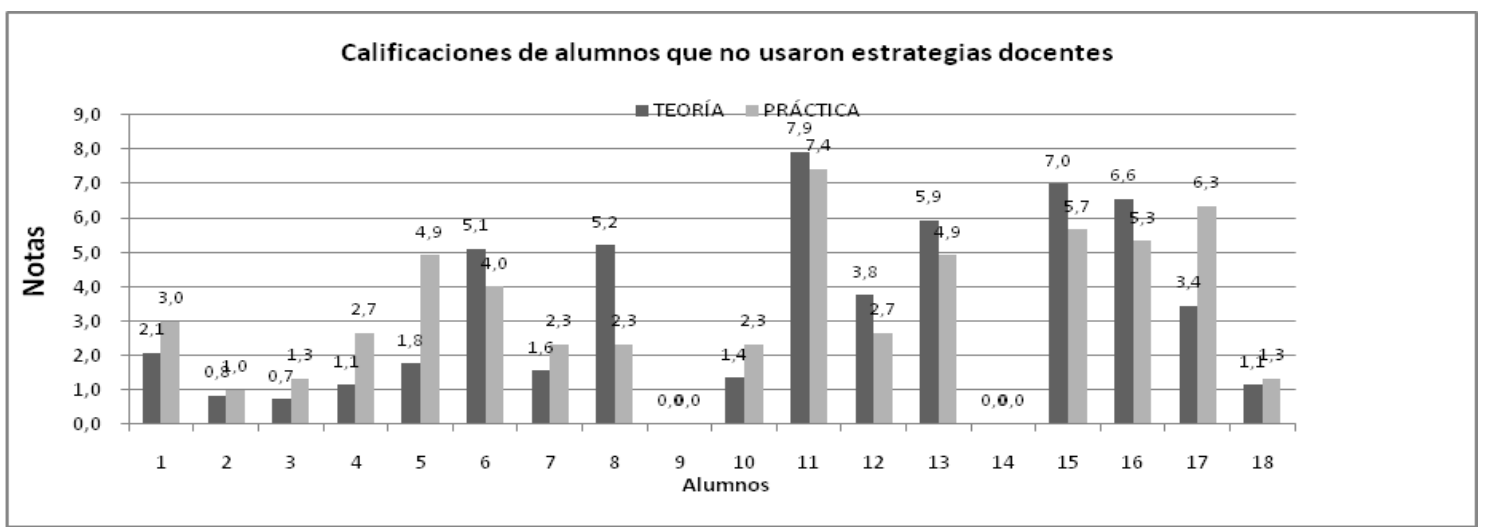

CUADRO 9. NúMERO de ALUMNOS Y PUNTUACIÓN OBTENIDA CON RESPECTO A LA TÉCNICA DEL PAPEL AL MINUTO

\begin{tabular}{|c|c|c|c|c|c|c|c|c|c|}
\hline \multirow[t]{2}{*}{ PREGUNTA } & \multicolumn{8}{|c|}{$\begin{array}{l}\text { Escala de puntuación } \\
\text { [0 (mínimo)- } 2 \text { (máximo)] }\end{array}$} & \multirow{2}{*}{$\begin{array}{l}\text { Media aritmética } \\
\text { ponderada } \\
\text { (puntuación) }\end{array}$} \\
\hline & 0 & 0,5 & 0,75 & 1 & 1,25 & 1,5 & 1,75 & 2 & \\
\hline $\begin{array}{l}\text { Cómo se registran los } \\
\text { deterioros en los activos } \\
\text { financieros mantenidos } \\
\text { para negociar }\end{array}$ & 8 & 3 & 2 & 2 & 4 & 2 & 2 & 4 & 0,90 \\
\hline $\begin{array}{l}\text { Diferencias entre permutas } \\
\text { comerciales y no } \\
\text { comerciales según el } \\
\text { PGCE }\end{array}$ & 9 & 4 & 3 & 4 & 2 & 1 & 1 & 3 & 0,74 \\
\hline $\begin{array}{l}\text { Diferencia entre valor } \\
\text { razonable y valor neto } \\
\text { realizable }\end{array}$ & 9 & 3 & 7 & 6 & 1 & 0 & 0 & 1 & 0,59 \\
\hline $\begin{array}{l}\text { ¿Qué es el fondo de } \\
\text { comercio? ¿Cómo se } \\
\text { contabiliza en el caso que } \\
\text { sea negativo? }\end{array}$ & 18 & 3 & 2 & 3 & 1 & 0 & 0 & 0 & 0,27 \\
\hline
\end{tabular}

(Dos preguntas de teoría realizadas en la convocatoria del examen de junio)

\footnotetext{
${ }^{14}$ Se usó la siguiente valoración numérica: cada pregunta valía 2 puntos, entre una escala de 0 (puntuación mínima) a 2 (puntuación máxima). Igualmente debajo de cada columna con la calificación obtenida y para cada cuestión, se indica el número de estudiantes que la consiguieron.
} 
Diferenciando los resultados anteriores, entre aquellos alumnos que asistieron a clase regularmente y los que no, es decir, los que utilizaron más asiduamente las estrategias del "papel al minuto", se pudo comprobar que un $40 \%$ de los estudiantes que asistió con regularidad, superó la parte teórica, por un 33\% que también superó dicha parte, pero sin presencia asidua a las clases. La diferencia, aunque con porcentajes menores, se sigue manteniendo levemente, en los resultados de la parte práctica, en las que el $30 \%$ de los encuestados que fueron regularmente a clase, obtuvieron mejores calificaciones, que aquellos que menos aparecieron por las aulas (28\%)15. Por lo general, del total de la calificación asignada en los exámenes de Contabilidad Financiera, suele ser en mayor porcentaje adjudicado a la parte práctica que a la teórica ${ }^{16}$, aunque para ser consecuente, la no asistencia a clase no debería haber condicionado la calificación final, puesto que el conjunto de preguntas formuladas por el docente, para la aplicación de esta técnica del papel al minuto, podría ser de dominio público, al haberlas transmitido los asistentes habituales a las clases, al resto de compañeros. Lo que sí parece desprenderse de los resultados, es que estas técnicas son más útiles para la parte teórica, tal y como ellos manifestaron en la encuesta los estudiantes.

La posibilidad de permitir el uso de un trabajo síntesis en la prueba final, al objeto de ayudar a la contestación de una pregunta de teoría, obtuvo un resultado bastante pobre, ya que sólo un $11 \%$ contestó la pregunta correctamente, por un $89 \%$ que, 0 bien, contestó equivocadamente, o la dejó en blanco. Una explicación a este resultado puede ser debido, a que el esfuerzo llevado a cabo para la realización del trabajo, no compensa la posibilidad de obtener un alto porcentaje de nota final, como efectivamente así ocurrió. Lo que sí se pudo observar, es que todos los alumnos que contestaron correctamente la pregunta, habían elaborado el trabajo de síntesis.

Al igual que se contempló con las técnicas del papel al minuto, se continúa con la misma tendencia a la hora de discriminar, entre aquellos alumnos que elaboraron los trabajos síntesis del temario de la materia, en función de las calificaciones observadas en el examen de junio, pudiendo advertirse, que el $30 \%$ de los estudiantes que entregaron los resúmenes, superaron la asignatura, por un $28 \%$ que también la superó, pero que no cumplieron con la tarea asignada. Estos resultados mejoran los éxitos obtenidos, tanto en la parte teórica como práctica del examen final, con un nivel de aprobados del $40 \%$ para los alumnos que elaboraron los resúmenes, por un $34 \%$ de estudiantes que superaron la parte teórica, sin haber presentado dichos trabajos. Esta tendencia se mantiene para la parte práctica en una proporción de $30 \%$ y $28 \%$ respectivamente. En definitiva y a tenor de los resultados, se puede comprobar la misma pauta que la obtenida para las técnicas del papel al minuto, en las que se aprecian, aunque levemente, mejores resultados para los alumnos que realizaron los trabajos síntesis que aquellos que no los presentaron.

Como se observa en el Gráfico 7, la valoración de la experiencia del trabajo en equipo, tampoco confirma buenos resultados respecto al uso de esta técnica docente. Del total de alumnos que hicieron el ejercicio práctico propuesto, relacionado con la preparación del tema mediante esta estrategia, sólo un 19\% logró superar satisfactoriamente dicho ejercicio ${ }^{17}$

\footnotetext{
15 Los resultados de la parte práctica presentan la misma tendencia respecto al número de alumnos que superó globalmente la materia, tal y como se puede observar en los Gráficos 5 y 6 .

16 En nuestro caso, así fue, ya que del $100 \%$ de la calificación final, el 55\% de la nota correspondió a la parte práctica.

17 Para una escala de puntuación entre 0 y 10, a efectos de calificar el ejercicio práctico propuesto, sólo un 7\% realizó el mismo correctamente. El mayor grupo de alumnos, se concentra en aquellos que obtuvieron entre 3 y 4 puntos, es decir, el $41 \%$ de los presentados al examen.
}

educade, no 2, 2011, p. 24 
Con el fin de ofrecer mayor rigor científico y poder comparar la proporción de aprobados en función del uso de estas metodologías, y dado que la muestra fue muy pequeña, sería necesario aplicar el "test exacto de Fisher"18.

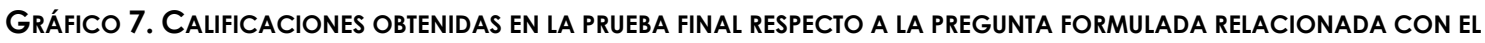
TRABAJO EN GRUPO

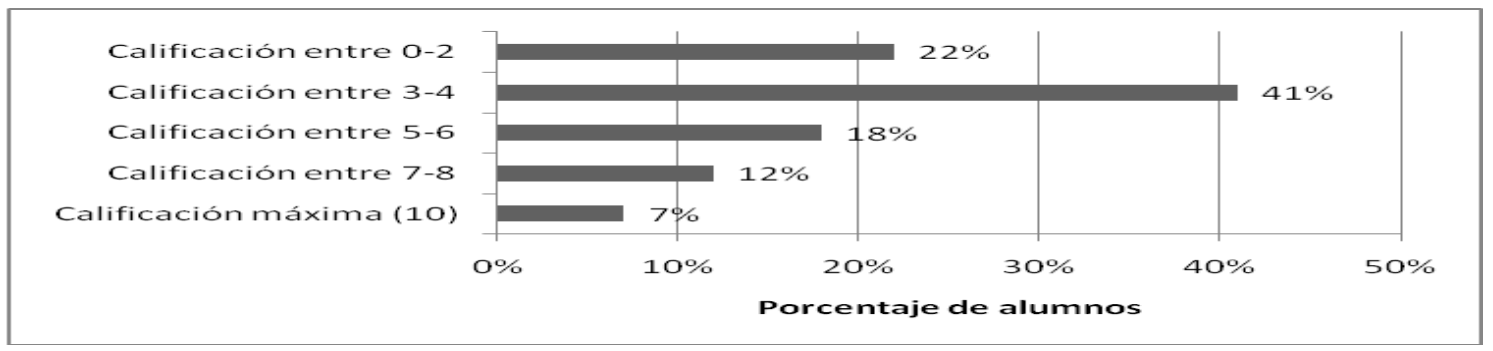

En primer lugar consideró los alumnos que habían seguido en conjunto las estrategias docentes (10 alumnos), frente a aquellos estudiantes que no las siguieron (18 alumnos). En el primer grupo el porcentaje de aprobados fue del $30 \%$ frente al $27,77 \%$, al aplicar en esa situación el test de Fisher, obtenemos un p-valor próximo a la unidad, con lo que no hay evidencia significativa para rechazar la hipótesis nula de igualdad, en el comportamiento entre los dos grupos.

Idéntico resultado estadístico se obtiene, si aplicamos este método a los resultados en la teoría, a pesar de que si sólo tenemos en cuenta los aspectos teóricos, el porcentaje de aprobados entre los que han seguido estrategias docentes es del $40 \%$, frente al 33,33\% de los que no las usaran. En lo que respecta a la práctica, la distribución de aprobados en los dos grupos, coincide con la de la nota total en la materia. Utilizando los datos numéricos correspondientes a la nota final de ambos tipos de grupos, deberemos aplicar una técnica no paramétrica, dado el pequeño tamaño de la muestra. Para ello se aplicó el "test de la suma de rangos de Wilcoxon" para el que se obtiene un $\mathrm{p}$-valor de 0.7552 , lo que indica que no hay diferencias significativas entre las notas de ambos grupos, es decir la calificación mediana de los que han seguido estrategias docentes (2.43), no difiere estadísticamente de la calificación mediana de los que no han seguido dichas metodologías (2.87). Para el caso el caso de las puntuaciones obtenidas en la teoría se observa alguna diferencia entre los alumnos que utilizaron o no las citadas técnicas, aún así el p-valor obtenido (0.3252) indica que no se pueden calificar como diferentes estadísticamente.

Por tanto, se puede observar que el uso de las estrategias docentes no contribuyó definitivamente a mejorar las calificaciones de la asignatura de Contabilidad Financiera, observando leves diferencias entre ambos grupos de estudiantes (los que las usaron y los que no), aunque con poca significación estadística.

\section{CONCLUSIONES Y FUTURAS LINEAS DE INVESTIGACIÓN}

El uso de las técnicas docentes del papel al minuto, realización de "trabajos-resumen" y la participación en un "trabajo en equipo" son percibidas por los alumnos, como una relevante ayuda a la docencia impartida a través de la lección magistral, constituyendo un instrumento útil, al objeto de paliar el problema de entendimiento y falta de atención de los alumnos en las aulas, de modo que puedan mejorar su aprendizaje e incrementar su participación activa en las clases, incrementando de

\footnotetext{
18 Este test, basado en una distribución hipergeométrica, permite contrastar la hipótesis nula de un valor del "odds ratio" igual a 1, o lo que es lo mismo, parte de la suposición de que la proporción de aprobados es la misma en los dos grupos considerados, en función de si han seguido o no las estrategias docentes.
} 
paso, su motivación. No obstante, ese grado de satisfacción percibido por los estudiantes, no resulta determinante en los resultados académicos, a la hora de discriminar entre aquellos alumnos que utilizaron estas estrategias docentes, respecto a los que no, permitiendo apreciar pocas diferencias entre aquellos estudiantes que habían superado la asignatura de Contabilidad Financiera, usando todas las estrategias de aprendizaje, que respecto a los que no las usaron. Estos porcentajes mejoran muy levemente, al comprobar un mayor porcentaje de aprobados, para la parte teórica respecto a la parte práctica del examen, aunque no fueron estadísticamente significativos.

Estas técnicas, permiten mejorar sin duda, muchas de las habilidades del estudiante, haciéndole el proceso de enseñanza más atractivo, sin embargo no contribuyen a mejorar la competencia que consideramos más relevante, es decir la referente al conocimiento de la materia, a juicio de los resultados, lo cual pone en duda el uso de estas nuevas herramientas, si las queremos utilizar como fin, más que como medio o complemento de las tradicionales.

Por tanto y a tenor de los resultados, parece claro que herramientas tradicionales como podría ser la lección magistral, sigue siendo, a pesar de todo el vehículo idóneo de transmisión de conocimientos, si nos atenemos, al menos, al uso del cóctel de estrategias experimentadas en este trabajo, ya que las mismas, pueden ayudar a la consecución de diversas competencias que emanan del EEES, puesto que no parecen sumar más valor añadido a los estudiantes.

Como reflexión hemos de considerar que parte del éxito educativo, depende en un alto grado, de la actitud del estudiante hacia el proceso de enseñanza, aspiraciones, madurez, pasado formativo y personal, lo que puede ser una explicación, independientemente de lo que los estudiantes opinen, del poco tiempo de estudio individual que invierten los alumnos, es decir la falta planificación, dedicación y continuidad de trabajo individual fuera de clase.

Si tenemos en cuenta que a partir de ahora, el alumno es el eje central del proceso de enseñanza-aprendizaje, su actitud debe ser activa y ser consciente que para lograr las competencias y habilidades es imprescindible que se implique proactivamente en el proceso. Esa resistencia del alumno, hacia una enseñanza centrada en el aprendizaje, que aumenta la responsabilidad en el estudiante, puede ser objeto de futuros trabajos que exploren como motivar a los mismos para conseguir mejores resultados.

Una de las limitaciones del trabajo está circunscrita a que la experiencia docente no estaba inmersa en un entorno del EEES, en el que tanto la asistencia, como la calificación del alumnado se limitó al uso de criterios de evaluación tradicionales, a través de un examen final y no a posibles métodos de evaluación de competencias y habilidades más acordes con una estrategia de aprendizaje activo más autónomo y permanente. Ello podría llevar a valorar para futuros estudios la mejora del potencial de dichas estrategias, en el nuevo entorno de Bolonia, en orden a criterios de evaluación continua. Para lo cual y como propuestas de mejora de las mismas, sugerimos las referentes a la posibilidad de evaluar el esfuerzo realizado por los alumnos con una contraprestación, de modo que el esfuerzo realizado les fuera compensado con una mejora o incremento en la nota final, ya que el mero aprendizaje no les motiva, si detrás no hay una recompensa.

A nuestro juicio y de acuerdo al panorama contable actual, también se podrían abrir nuevas vías de investigación futuras encaminadas a la búsqueda de criterios de evaluación más eficientes, que puedan conjugar el aspecto de motivación del alumnado, al objeto de incrementar significativamente su rendimiento académico, sobre todo para las estrategias del papel al minuto y de los trabajos-resumen, éste último como instrumento de aprendizaje teórico por medio del trabajo individual del alumno. 


\section{BIBLIOGRAFÍA}

AlDAZABALL, E. Y M. ESPINOSA [2011]: Comportamiento y formación ética: percepción y expectativas de los estudiantes y profesores universitarios de contabilidad. XXI Jornadas Hispano-Lusas de Gestión Científica, ETEA, Córdoba. Tomo IV: 376391.

ANAYA NiETO, D. [2005]: Efectos del resumen sobre la mejora de la metacomprensión, la comprensión lectora y el rendimiento académico. Revista de Educación n 37: 281-294.

Anson, C. M., Bernold, L. E., Crossland, C., Spurlin, J., MCDermotr, M. A. y S. Weiss, S. [2003] Empowerment to Learn in Engineering: Preparation Foran Urgently-Needed Paradigm Shift. Global Journal of Engineering Education, 72): 145-155.

Argüelles Álvarez, I. [2004]: Evaluación y calificación de resúmenes de textos expositivos. Revista lbérica: 81-9.

ARQUERO J.L., DONOSO, J.A., HASSALL, T. Y J.JOYCE [2001]: Vocational skills in the accounting professional profile: The Chartered Institute of Management Accountants (CIMA) employers'opinion'. Accounting Education, 10, 3: 299-313

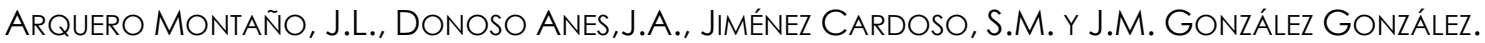
[2009]: Análisis exploratorio del perfil demandado para administración y dirección de empresas: implicaciones para el área contable. Spanish Accounting Review - Revista de Contabilidad. Vol. 12, 2

AusuBEL, D.P. [2002]: Adquisición y retención del conocimiento. Una perspectiva cognitiva. Paidós, Barcelona.

AZKUE IRIGOYEN, I. [2010]: Dilemas éticos como intervención formativa innovadora en la asignatura de Auditoría de Cuentas. VII Jornadas de Docencia en Contabilidad. ASEPUC, Bilbao.

BARALDÉs CAPDEVILA M. [2010]: Reflexiones sobre trabajo en grupo presencial versus trabajo individual on-line. Una propuesta de "aprendizaje mezclado". VII Jornadas de Docencia en Contabilidad. ASEPUC, Bilbao

BREWER, W. Y M.I. MendelSON, I. [2003]: Methodology and Metrics for Assessing Team Effectiveness. International Journal of Engineering Education, 19(6): 777- 787.

BROOKS, C. M. Y J.L. AMmONS, J. L. [2003]: Free Riding in Group Projects and the Effects Oftiming, Frequency, and Specificity of Criteria in Peer Assessments. Journal of Education for Business, 78(5): 268-272.

CANAY PAZOS, J.R. Y S. CANTORNA AGRA [2009]: LOS mapas conceptuales en contabilidad: Una herramienta para el EEES. VI Jornadas de Docencia en Contabilidad. ASEPUC, Sevilla.

CANTILLON, P. [2003]: ABC of learning and teaching in medicine, teaching large groups. British Medical Journal, 326-437

Carrasco, A., Donoso, J.A., Duarte, T., Hernández, J.J.lópez, R. y C. NúÑez [2009]: Aprendizaje basado en proyectos versus aprendizaje basado en actividades: Una experiencia en la elaboración y análisis de los estados financieros. La formación en contabilidad en los grados y másters. VI Jornadas de Docencia en Contabilidad. ASEPUC, Sevilla.

CAstrillo Lara, L. y M.M. Delgado HuRTAdo [2010]: El aprendizaje cooperativo en la enseñanza universitaria en Contabilidad. VII Jornadas de Docencia en Contabilidad. ASEPUC, Bilbao.

ChIZMAR, J.F. Y OSTROSKY, A.L. [1998]: The one-minute paper: some empirical findings, Journal of economic education, vol. 29, Num. 1, Winter, 1998.

Christoforou, A. P., Yigit, A. S., Al-AnSARY, M. D., Ali, F., Aly, A. A., LABABIDI, H., NASHAWI, I. S., TAYFUN, A. Y M. ZRIBI [2003]: Improving Engineering Education at Kuwait University Through Continuous Assessment. International Journal of Engineering Education, 19(6): 818-827. 
CONFERENCIA DE DECANOS DE FACULTADES DE CIENCIAS ECONÓMICAS y EMPRESARIALES (CONFEDE) [2005]: Libro blanco sobre los estudios de grado en economía y en empresa. En http://www.aneca.es/var/media/150292/libroblanco_economia_def.pdf

CROSS, K.P., Y ANGELO, T.A. [1993]: Classroom assessment techniques: A handbook for college teachers, Jossey-Bass, San Francisco.

CURÓs I VILÁ M.P. [2009]: Los alumnos en el aula. una experiencia de trabajo cooperativo en contabilidad. VI Jornadas de Docencia en Contabilidad de ASEPUC, Sevilla: 1-5.

DANCER, D. Y P. KAMvounias [2005]: Student Involvement in Assessment: a Project Designed to Assess Class Participation Fairly and Reliably. Assessment and Evaluation in Higher Education: An International Journal, 30(4): 445-454.

DíAZ B., F. Y R.G. HERNÁNDEZ [1999]: Estrategias docentes para un aprendizaje significativo. Una interpretación constructivista. McGrawHill, México. Capít. 5. En http://redescolar.ilce.edu.mx/redescolar/biblioteca/articulos/pdf/estrate.pdf: $1-27$

DRUMMOND, G. [2007]: Programme feedback: it only takes a minute! International Journal of Educational Management, Vol. 21 Iss: 1, pp.29 - 36.

FROYD, J. Y J. LAYNE [2008]: Faculty Development Strategies for Overcoming the"Curse of Knowledge". October 22 - 25, 2008, Saratoga Springs, NY 38th ASEE/IEEE Frontiers in Education Conference S4D-16 http://ieeexplore.ieee.org/stamp/stamp.jsp?tp=\&arnumber $=4720529$

Gómez Ruíz, L.M. Y D. NARANJO GIL [2010]: La competencia de trabajo en grupo en la asignatura de Contabilidad de Gestión: Una propuesta de actividad y de evaluación. VII Jornadas de Docencia en Contabilidad. ASEPUC, Bilbao

GraefF, T.R. [2010]: Strategic Teaching for Active Learning. Marketing Education Review, Vol. 20 Issue 3, p265-278

Holtham, C. W.; MelVILLE, R. R.; y M.S. SODHI [2006]: Designing Student Groupwork in Management Education: Widening the Palette of Options. Journal of Management Education, 30(6): 809-817.

INOUE, A. B. [2005]: Community-based assessment. pedagogy. Assessing Writing 9(3): 208238.

INTERNATIONAL FEDERATION OF ACCOUNTANTS [2009]: Handbook of international education pronouncements. En http://www.ifac.org

JenkINS, H. Y L.W. LACKEY [2005]: Preparing Engineering Students for Working in Teams Through Senior Design Projects. IEEE International Professional Communication Conference Proceedings.

Kalliath, T. Y M. LAIKEN [2006]: Use of Teams in Management Education. Journal of Management Education, 30(6): 747-750.

LEEMING, F.C. [2002]: The Exam-A-Day Procedure Improves Performance in Psychology Classes. Teaching of Psychology, vol. 29, n 3, 210-212

León Sendra, A. Y L. García Magald [2010]: El "one minute paper" como elemento eficaz y asequible para la mejora de la calidad docente en el espacio europeo de educación superior. Actas I Jornadas Andaluzas de Formación Inicial del Profesorado Universitario. Universidad de Granada

LeRís LÓPEZ,M. D. y M.L. SeIn EChAlUCe LACLETA [2009]: Una experiencia de innovación docente en el ámbito universitario. Uso de las nuevas tecnologías. Arbor, CLXXXV: 93-110.

LLORET, J. y J.A. MARIN García [2007]: Novel and Stable Lecturers' Point of View About University Students Working Groups. In P. Dondon, et al., Mathematical Methods and Computational Techniques in Research and Education, (p. 368373). Arcachon, France: WSEAS Press.

LUCAS, G.M. [2010]: Initiating Student-Teacher Contact Via Personalized Responses to One Minute Papers. College Teaching, Vol. 58 Issue 2, 39-42

MARCOS VARGAS [2009]: Papeles de trabajo. Cátedra metodología de la Investigación I. En www.no0b2hack.com.ve/descargas/r.pdf : 1-5. 
Marín García,J.A., Millares Insa, C., García Sabater, J.J. Y P.I.Vidal Carreras [2008]: Enseñando Administración de Empresas con docencia basada en el trabajo en equipo de los estudiantes: Ventajas, inconvenientes y propuestas de actuación. Intangible Capital, Vol. (4)2,: 143-164.

MICHAELSON, R., [2003]: Assessing Group Work. Briefing paper for LTSN-BEST. http://www.business.heacademy.ac.uk/publications/misc/briefing/groupwork/ assessing\%20group\%20work\%20-\%20michaelson.pdf.

Ministerio DE EDUCACIÓN y ClenCIA [2006]: Propuestas para la renovación de las metodologías educativas en la Universidad. En http://www.unirioja.es

Morales Vallejo, P. [2011]: El one minute paper. Universidad Pontificia Comillas, Facultad Ciencias Humanas Madrid. http://www.upcomillas.es/personal/peter/otrosdocumentos/OneMinutePaper. pdf

MOReno Ramón, H., IBAÑeZ ASensio, S. Y J.M. GIsBert Blanquer [2009]: Autonomía y tutorización como base del aprendizaje en asignaturas de Génesis de Suelos. A 'Taula B: Autogestió de l'aprenentatge'. Girona: Universitat. Disponible en: http://hdl.handle.net/10256/1997

Noguera C. [2009]: Técnicas de estudio y estrategias de aprendizaje. Capítulo 15, en http://www.mailxmail.com/curso-tecnicas-estudio-estrategiasaprendizaje/tecnicas-resumen-sintesis-resenas.

Project Designed to Assess Class Participation Fairly and Reliably. Assessment and EVAluation IN HIGHER EDUCATION: An International Journal, 30(4): 445-454.

RAWSON, K.; DUNLOSKY, J Y K.W. THIEDE [2000]: The rereading effect: Metacom-prehension accuracy improves across reading trials. Memory and Cognitton, 28: 10041010.

RAWSON, K Y J. DUNLOSKY [2002]: All performance prediction for text based on case of processing? Journal of Experimental Psychology: Learning, Memory, and Cognition, 26: 69-80.

RIVero Menéndez, R., Rivero Menéndez, J.A. Y L. Gil HerRera [2000]: El "one minute paper" y algunas otras técnicas docentes. IX Encuentro de ASEPUC, Las Palmas de Gran Canaria 24-26 de mayo: 1-10.

Rodríguez MOlinuevo, J.M. [2010]: Nuevos retos en la formación de los futuros profesionales contables. VII Jornadas de Docencia en Contabilidad. ASEPUC, Bilbao.

Rugarcia, A., Felder, R. M., Woods, D. D. y J.E. Stice [2000]: The Future of Engineering Education: A Vision for a New Century. Chemical Engineering Education, 34(1): 16-25.

Sáez Ocejo, J.L., Beltrán Varandela, J.L. e I. Garrido Valenzuela [2010]: La estrategia docente del papel al minuto aplicada a materias del área de economía financiera y contabilidad dentro del Espacio Europeo de Educación Superior. Global Financial \& Business Networks and Information Management Systems. Coord. I.A. Maximsev, V.V. Krasnoproshin y C.Prado Román.

SÁez OCEJo, J.L. Y M.P. Rodríguez Rodríguez [2003]: Una nueva técnica docente. El punto más dudoso. Comunicación a la Asociación Española de Economía de la Empresa. XIII Jornadas Hispano-Lusas de Gestión Científica, Lugo, 12-14 de febrero.

SÁez Ocejo, J.L. Y M.P. Rodríguez Rodríguez [2004]: Estudio empírico de la técnica de evaluación del papel al minuto en Contabilidad Financiera. Comunicación presentada a las IV Jornadas de Docencia en Contabilidad: Sevilla.

SHEPPARD, K., DOMINICK, P. Y Z. ARONSON [2004]: Preparing Engineering Students for the New Business Paradigm of International Teamwork and Global Orientation. International. Journal of Engineering Education, 20(3): 475-483.

STEAD, D.R. [2005]: A review of the one-minute paper. Active Learning in HigherEducation, Vol. 6, No. 2, 118-131. 
Watts, F., García Carbonell, A. Y J. LloRens [2006]: Introducción a la Evaluación Compartida: Investigación Multidisciplinar. En Watts, F. y García-Carbonell, A., La Evaluación Compartida: Investigación Multidisciplinar (p. 1-9), Valencia: Editorial de la UPV.

WILLCOXSON, L. E. [2006]:"It's Not Fair!": Assessing the Dynamics and Resourcing of Teamwork. Journal of Management Education, 30(6): 798-808.

YOUNG, C. B. Y J.A. HENQUINET [2000]: A Conceptual Framework for Designing Group Projects. Journal of Education for Business, 76(1): 56-60.

ZelLIK, M. [2010]: Classroom Assessment Techniques Minute Paper. Webpage Department of Physics \& Astronomy University of New Mexico. Disponible en: http://www.flaguide.org/cat/minutepapers/minutepapers6.php

ZIPITRIA, I.; ELORRIAGA, J.A. Y A. ARRUARTE [2007]: Hacia la automatización de la evaluación de resúmenes desde la experiencia cognitiva. Revista Iberoamericana de Información Educativa $n^{\circ}$ 5, enero-junio: 49-61 
José Luis Beltrán, José Manuel Pereira, José Luis Sáez

Aplicación práctica de técnicas docentes para Contabilidad Financiera

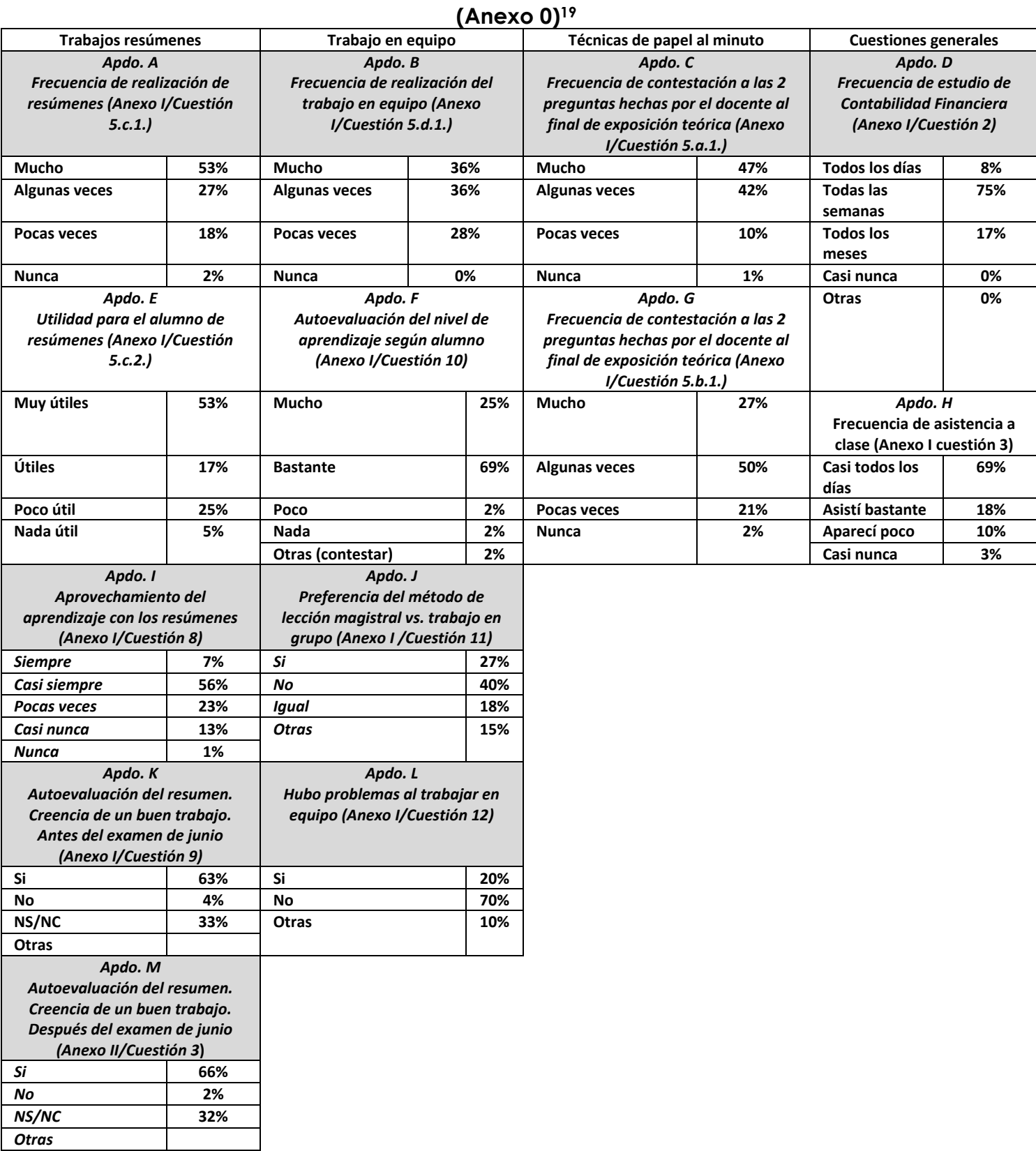

19 En la presente tabla resumimos otras estadísticas para una mejor apreciación de los resultados del estudio, para ello hemos dividido la misma en cuatro conceptos (por columnas) y cada columna, a su vez, los subdividimos en apartados (del A al M). En el título de cada apartado, se hace referencia a los Anexos I y II de los cuestionarios completados por los alumnos, en donde también se detallan, los números de ítem-cuestión, de dichos cuestionarios, para su mejor identificación. 
(Anexol)

\section{CUESTIONARIO SOBRE LA TÉCNICAS DOCENTES USADAS EN EL CURSO 2009-2010 EN CONTABILIDAD FINANCIERA I $\left(1^{\circ} \mathrm{B}\right)$}

1) ¿Crees que esta asignatura debe estudiarse llevándose al día?
$\square \mathrm{Si}$
$\square$ No
Otras (explicar brevemente)

2) ¿Con qué frecuencia estudias esta asignatura?:

Todos los días $\square$ Todas las semanas $\square$ Todos los meses $\square$ Casi nunca $\square$ Otras (indicar)

3) Has venido a clase con frecuencia (Indicar frecuencia)
Casi todos los días
Asistí bastante
Aparecí poco
Casi nunca

4) ¿Eres consciente si el profesor ha utilizado algunas técnicas docentes (o métodos u otros medios) para aprender la asignatura?

$$
\text { Si } \square \text { No }
$$

5) En caso de contestar sí a la respuesta anterior indicar cuales has utilizado (indicando a su vez la frecuencia de uso y la utilidad para aprender la asignatura desde tu punto de vista):

a. 2 preguntas que nos hacían después de las clases de teoría y que debíamos contestar

1. $\square$ Mucho $\square$ Algunas veces $\square$ Pocas veces $\square$ Nunca lo use

2. $\square$ Muy útil $\square$ Útil $\square$ Poco útil $\square$ Nada útil

b. Dudas que podía indicar por escrito al profesor en el papel-cuartilla que me pedía (por la parte de atrás de las preguntas)

1. $\square$ Mucho $\square$ Algunas veces $\square$ Pocas veces $\square$ Nunca lo use

2. $\square$ Muy útil $\square$ Útil $\square$ Poco útil $\square$ Nada útil

c. Realización de resúmenes de los temas del libro de teoría

1. $\square$ Mucho $\square$ Algunas veces $\square$ Pocas veces $\square$ Nunca lo use

2. $\square$ Muy útil $\square$ Útil $\square$ Poco útil $\square$ Nada útil

d. Realización de un trabajo en grupo, posteriormente expuesto en clase ante el profesor y compañeros

1. $\square$ Mucho $\square$ Algunas veces $\square$ Pocas veces $\square$ Nunca lo use

2. $\square$ Muy útil $\square$ Útil $\square$ Poco útil $\square$ Nada útil 
6) En caso de contestar "Poco útil" o "Nada útil", en la pregunta anterior (5:a,b,c,d) para alguna de las respuestas anteriores, explicar brevemente porqué, indicando la técnica en cuestión

\begin{tabular}{|c|c|}
\hline $\begin{array}{l}2 \text { preguntas que nos hacían } \\
\text { después de las clases de teoría } \\
\text { y que debíamos contestar }\end{array}$ & \\
\hline $\begin{array}{l}\text { Dudas que podía indicar por } \\
\text { escrito al profesor en el papel- } \\
\text { cuartilla que me pedía (por la } \\
\text { parte de atrás de las } \\
\text { preguntas) }\end{array}$ & \\
\hline $\begin{array}{l}\text { Realización de resúmenes de } \\
\text { los temas del texto básico }\end{array}$ & \\
\hline $\begin{array}{l}\text { Realización de un trabajo en } \\
\text { grupo, } \\
\text { expuesto en clase ante el } \\
\text { profesor y compañeros }\end{array}$ & \\
\hline TÉCNICA & ¿PORQUÉ LA CONSIDERO “POCO” ÚTIL O “NADA” ÚTIL? \\
\hline
\end{tabular}

7) ¿El profesor contestó a las preguntas formuladas en la sesión posterior, sobre las 2 preguntas formuladas en la en clase, en la sesión previa?
Siempre
Casi siempre
Pocas veces
Casi nunca
Otras (indicar la respuesta)

8) ¿Consideras que los trabajos resumen, por ti realizados de los temas de la asignatura, te han valido para aprender la misma?
Siempre
Casi siempre
Pocas veces
Casi nunca
Otras (indicar la respuesta)

9) ¿Consideras que has hecho unos buenos resúmenes?
Si $\square$ No
No sabe/No contesta nunca
Otras (indicar la respuesta)

10) ¿Aprendiste los temas, con el trabajo realizado en grupo y que entregaste al profesor en power- point? (cuentas de personal y tesorería)
Mucho
Bastante
Poco
Nada
Otras (indicar la respuesta)

11) ¿Hubieras preferido que el profesor te los hubiese explicado en clase, como hace habitualmente?
$\mathrm{Si}$
No
Me daría igual
Otras (indicar la respuesta)

12) ¿Tuviste problemas al trabajar en grupo con otros compañeros, en la elaboración del trabajo en power- point (cuentas de personal y tesorería)?

$$
\text { Si } \square \text { No } \square \text { Otras (indicar la respuesta) }
$$

13) ¿Hubieras preferido hacerlo sólo el trabajo en power- point? (cuentas de personal y tesorería)

$$
\text { Si } \square \text { No } \square \text { Me daría igual } \square \text { Otras (indicar la respuesta) }
$$

14) ¿Puedes indicarme algún aspecto que mejore estas actividades o darme alguna idea 0 comentario sobre cómo mejorar estas técnicas docentes, o consejos para mejorar la docencia o incluso sugerir alguna tú? 


\section{(Anexo II)}

CUESTIONARIO SOBRE LA TÉCNICAS DOCENTES USADAS EN EL CURSO 2009-2010 EN CONTABILIDAD FINANCIERA I ( $\left.1^{\circ} \mathrm{B}\right)$ - (el día del examen de junio)

1) El examen se ha ajustado a las indicaciones apuntadas por el profesor en clase en cuanto a la forma del mismo

$$
\square \text { Si } \square \text { No } \square \text { Otras (indicar la respuesta }
$$

2) ¿Consideras que los trabajos resúmenes, por ti realizados de los temas de la asignatura, te han valido para aprender la misma?

$$
\square \text { Siempre } \square \text { Casi siempre } \square \text { Pocas veces } \square \text { Casi nunca } \square \text { Otras (indicar la respuesta) }
$$

3) ¿Consideras que has hecho unos buenos resúmenes, una vez hecho el examen?

$$
\square \text { Si } \square \text { No } \square \text { No sabe/No contesta nunca } \square \text { Otras (indicar la respuesta) }
$$

4) ¿Te valió de algo cara a realizar el examen las 2 preguntas formuladas por el profesor que tenías que contestar al final de cada sesión teórica?

$\square$ Si $\square$ No $\square$ Poco $\square$ Otras (indicar la respuesta) 